\title{
HISTOIRE DES FEUX ET PRATIQUES AGRAIRES DU NÉOLITHIQUE À L'ÂGE DU FER EN RÉGION CENTRE
}

\section{Implications territoriales, démographiques et environnementales}

\author{
Boris VANNIÈRE* et Rémi MARTINEAU*
}

\begin{abstract}
Mots-clés. Microcharbon de bois, feu, défrichements, pratiques agraires, Néolithique, Grand-Pressigny, Protohistoire, Berry.
Résumé. La question du développement des pratiques agraires, du Néolithique à l'âge du Fer, est posée à partir d'une étude paléoenvironnementale réalisée en région Centre. Celle-ci repose sur une reconstitution des dynamiques sédimentaires et de l'histoire des feux. Durant la première partie de l'Holocène, jusqu'au Néolithique ancien, le régime des feux semble essentiellement contrôlé par les conditions climatiques; les ouvertures du milieu associées aux feux ont pu alors favoriser les implantations humaines. Au cours du Néolithique ancien et final, au début et à la fin de l'âge du Bronze et au début de l'âge du Fer, les occurrences de feux et les phénomènes d'érosion pourraient être en partie d'origine anthropique et correspondre à la mise en place et au développement des systèmes agraires pré-et protohistoriques. I. a simullanéité des occurrences de feux de grande ampleur et des importantes productions laminaires en silex du Grand-Pressigny attestent une modification des pratiques agraires et peut-être une expansion territoriale importante, en réponse aux variations paléodémographiques liées aux besoins alimentaires. Les différences observées dans l'intensité de l'influx en charbon de bois et dans les formes d'érosion du Néolithique et de l'âge du Fer reflètent également une évolution dans le système agraire. Les sociétés sont progressivement passées d'un système agraire à culture sur abattis-brûlis itinérant à un système agraire " post-forestier ", aver jachère et élevage pastoral associé. A u cours de l'âge du Bronze, les périodes de dégradation des conditions climatiques sont sỵnchrones d'une déprise agraire et/ou d'une modification importante des systèmes économiques et sociaux qui vont entraîner une modification durable des systèmes agraires. Les similitudes observées avec d'autres régions françaises et européennes impliquent que les phénomènes observés sont de grande ampleur et s'inscrivent dans une dynamique évolutive générale de longue durée, soumise aux facteurs communs que sont la démographie et le climat.
\end{abstract}

Key-words. Micro-charcoal, fire, clearance, farming practices, Neolithic, Grand-Pressigny, Protohistory, Berry (France).

Abstract. Fire history and land use practices from the Neolithic to the Iron Age in central France: territorial, demographic and environmental implications. Archaeological and palaeoenvironmental studies have been carried out in central France, aiming at the reconstruction of farming activities from the Neolithic to the Iron Age, most particularly during the Late Neolithic. Data obtained are based mainly on sedimentary dynamics and fire history. During the first part of the Holocene, until the Early Neolithic, fire occurrence is largely controlled by climatic conditions. These may have favoured human activities, especially land occupation and use. Fire and erosion events recorded during the Middle-Late Neolithic, the Late Bronze Age and the early Iron Age appear as antropogenically induced (at least partly); they correspond to the establishment and development of pre- and protohistoric agricultural systems. During the Late Neolithic,

* UMR 6565 "Laboratoire de Chrono-écologie ", CNRS - U'niersité de Franche-Comté, LFR Sciences et techniques, 16 route de Gray, F-25030 Besançon. Courriel : boris.vamniere@ ${ }^{\circ}$ univ-fcomte.fr.

** UMR 5594 "Archéologie, cultures et sociétés ", CNRS - Liniversité de Bourgogne - Ministère de la Culture, 6 boulevard Gabricl, 21000 F-Dijon. Courriel : remi.martineau@u-bourgogne.fr. 
between 3200 and $2400 \mathrm{cal}$. BC, the occurrence of large simultaneous fires and the intensive production and exportation over the northeastearn Europe of flint implements from the Crand-Pressigny area (Indre-et-Loire, France) testify to changing farming practices and colonization of new land as a result of new dietary needs and demographic pressure. Variable rates of charcoal influx and erosion processes also reflect the evolution of the agricultural system. From the Neolithic to the Iron Age, slash-and-burn s slowly replaced by a "post-forest" system, based on the practice of fallowing land and animal husbandry. The same appears to happen elsewhere, both in France and in other European countries; this implies that phenomena observed are not local and reflect a general long term evolution based on common factors, such as climate and demography.

Schlagwörter. Holzkohleanalyse, Feuer, Rodungen, Agrarwirtschaft, Neolithikum, Grand-Pressigny, Prähistorische Metallzeiten, Berry (Frankreich).

Zusammenfassung. Anhand einer in der Region Centre durchgeführten Untersuchung zur Paläoumwelt, die auf einer Rekonstruktion der Sedimentdynamik und der "Geschichte des Feuers" beruht, fragen wir nach der Entwicklung der Agrarwirtschaft vom Neolithikum bis in die Eisenzeit. Während eines ersten Abschnitts des Holozän, nämlich bis zum Frühneolithikum, scheint das Feuerregime im wesentlichen von den Klimabedingungen gesteuert. Öffnungen der Vegetation, wie sie mit Bränden einhergehen, konnten in dieser Zeit menschliche Niederlassungen begünstigen. Im Verlauf des Früh-und des Spätneolithikums, am Anfang und am Ende der Bronzezeit sowie am Anfang der Eisenzeit könnten das Auftreten von Bränden und die Erosionsphänomene dagegen zumindest teilweise auf menschliches Wirken zurïckgehen und der Einrichtung und Entwicklung vor- und frühgeschichtlicher Agrarsysteme entsprechen. Das gleichzeitige Auftreten großer Brandereignisse und von umfangreichen Klingenproduktionen in Grand-Pressigny-Silex bezeugt einen agrarwirtschaftlichen Wandel und unter Umständen auch eine starke territoriale Expansionsbewegung, die als Antwort auf paläodemographische Schwankungen in Abhängigkeit vom Ernährungsbedarf zu sehen wären. Die an Vergleichswerten des Neolithikums und der Eisenzeit beobachteten Unterschiede beim Holzkohleeintrag und bei den Erosionsformen spiegeln ebenfalls eine Entwicklung des Agrarsystems wider: Die prähistorischen Siedelgruppen gingen schrittweise vom System eines Brandrodungswanderfeldbaus zu dem einer Bewirtschaftung der gerodeten Waldbrachen mit Beweidung durch Herden über. Während der Bronzezeit entsprechen die Zeiten der Klimaverschlechterung einer Periode des Niedergangs der Agrarwirtschaft und/oder beträchtlichen Veränderungen der Wirtschafts- und Gesellschaftsordnung, die letztlich eine dauerhafte Veränderung der Agrarsysteme zur Folge haben. Die Ähnlichkeiten zu den Verhältnissen in anderen Regionen Frankreichs und Europas lassen darauf schließen, daß die beobachteten Erscheinungen weit ausgreifen und an der Gesamtdynamik einer Langzeitentwicklung Anteil haben, die den gemeinsamen Faktoren Demographie und Klima unterworfen ist.

Übersetzung: Stefan WIRTH

Le rôle de l'homme dans la transformation durable des écosystèmes est aujourd'hui au centre des débats de notre société. Connaître et comprendre le développement des systèmes agraires pré- et protohistoriques et leur relation avec les modifications écologiques et climatiques peut apporter une perception de la longue durée. Vision utile à la fois aux gestionnaires actuels du patrimoine environnemental et aux archéologues pour une meilleure analyse du développement des sociétés passées. Des débuts de l'agriculture au Néolithique jusqu'à l'âge du Fer, les conditions et les modalités de mise en place et de développement des systèmes agraires en France sont encore mal connues, alors que ces systèmes constituent certainement les pivots des interactions entre les sociétés humaines et leur environnement (Guilaine dir., 1991 ; Mazoyer, Roudart, 1997 ; Guilaine, 2000). Les études archéologiques des habitats lacustres des Alpes du Nord, du Jura suisse et français, couplées avec différentes approches paléoclimatiques et paléobotaniques ont permis de proposer des schémas systémiques alliant amélioration climatique, baisse du niveau des lacs, production agricole et développement démographique au cours du Néolithique final (Arbogast et al., 1996 ; Pétrequin et al., 1998). Ces travaux insistent à la fois sur l'importance des paramètres environnementaux dans l'évolution des sociétés mais aussi sur les capacités qu'ont ces sociétés à modifier leurs systèmes de production en fonction de leurs besoins liés à leur évolution démographique. Cependant de telles études restent aujourd'hui rares et centrées sur des espaces géographiques très localisés et des écosystèmes très particuliers.

Cette étude s'intéresse à un vaste plateau calcaire en bordure sud du Bassin parisien, la Champagne berrichonne en Berry (fig. 1). Cette zone de basse altitude reste encore peu documentée en ce qui concerne les relations entre sociétés, écologie et climat (Buchsenschutz et al., 1988 ; Guilaine, 1997 ; Batardy et al., 2001 ; Vannière, 2001). 


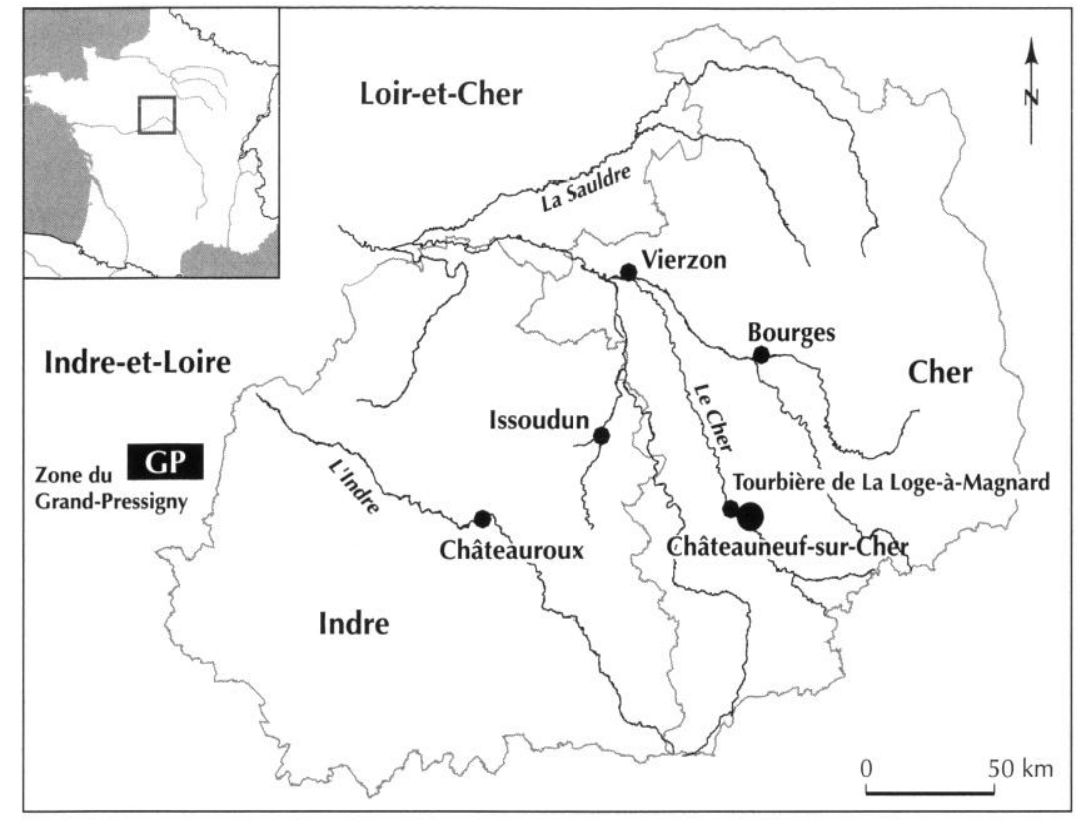

Fig. 1 - Localisation de la zone d'étude : région Centre. Localisation de la zone du CrandPressigny, de la tourbière de La Loge-à-Magnard (Chavannes, Cher) et des principaux sites archéologiques mentionnés dans le texte.
Au cours de l'histoire européenne, essartage, écobuage, brûlage et feu pastoral ont constitué des aspects essentiels de l'écosystème cultivé et pâturé (Sigaut, 1975 ; Métailié, 1981 ; Vannière et al., 2003). Le feu a aussi été largement évoqué comme un des principaux outils de la transformation du milieu pour les défrichements ou l'entretien des espaces agropastoraux durant la Préhistoire (Iversen, 1941 et 1949 ; Mazoyer, Roudart, 1997 ; Vannière, 2001). Dans les forêts tempérées du nord de l'Europe, depuis le Néolithique jusqu'au Moyen Âge, la prédominance d'un système de culture sur abattis-brûlis (slash-and-burn) est démontrée (Iversen, 1956 ; Huttunen, 1980 ; Vuorela, 1986 ; Pitkänen, Huttunen, 1999). Plus au sud, en montagne pyrénéenne, de récentes études paléoenvironnementales attestent que le feu a été utilisé par les communautés du Néolithique pour des défrichements (Vannière et al., 2001 ; Galop et al., 2002). Le feu anthropique, ou plus précisément les traces qu'il laisse, par la production de très nombreux microcharbons de bois, peut représenter un marqueur essentiel de la transformation du milieu, directement lié à la mise en valeur agricole (Vannière, 2004). Les reconstructions des occurrences des feux et leur quantification représentent un potentiel intéressant pour l'étude des processus et rythmes de l'anthropisation (Morison, 1994 ; Moore, 2000). Par ce moyen, il s'agit alors de reconstituer les chronologies des grands défrichements préhistoriques. Le développement des études sur l'histoire des feux se justifie d'autant plus que les marqueurs stratigraphiques de l'action de l'homme sur le milieu, hors contexte archéologique, comme les Indices polliniques d'anthropisation (IPA) sont rares (Richard, 1995). Cependant, si de nombreux auteurs attestent l'occurrence des feux au cours de l'Holocène et leur rôle dans la transformation des écosystèmes, l'origine de ces feux reste encore une question vive dans la plupart des cas (Clark et al., 1989 ; Richoz et al., 1994 ; Carcaillet, 1998 ; Innes, Simmons, 2000 ; Vannière, Laggoun-Defarge, 2002).

Ce travail présente l'étude paléohydrologique et l'étude du signal incendie, en termes d'origine, d'occurrence et d'ampleur des feux, d'une séquence sédimentaire couvrant une période de près de 5000 ans, comprise entre 5500 et 700 cal. BC. Il s'agit dans un premier temps de proposer une reconstruction des évolutions paléoenvironnementales sur la longue durée et de montrer la place des feux dans ces évolutions. L'origine des feux, incendies climatiques et feux anthropiques, sera ensuite discutée en relation avec les évolutions du climat et les sociétés humaines, en essayant d'évaluer leur impact sur l'environnement ainsi que les éventuels "déterminismes " environnementaux sur les communautés humaines. L'objectif général est d'apporter un certain nombre d'éléments de réponse sur les interactions entre les évolutions respectives du climat, des systèmes agraires (type de pratique) et des sociétés, du Néolithique à l'âge du Fer. L'accent sera également mis sur l'apport des analyses de l'histoire des feux à l'interprétation du "phénomène Grand-Pressigny » (Mallet, 1992). 
L'hypothèse de départ est qu'une estimation de l'importance des défrichements et de l'ouverture du milieu par l'homme peut apporter une image de la dimension des occupations humaines et des variations démographiques. La stratégie d'étude consiste à rechercher des indices de modification des activités humaines conjointement dans l'environnement et dans la culture matérielle des sociétés et à les mettre en relation dans un système global où interagissent le climat, l'environnement naturel, le système agraire, la production et la fonction des outils, l'alimentation et la démographie.

\section{CONTEXTE ET PROBLÉMATIQUES ARCHÉOLOGIQUES}

La région Centre (fig. 2) offre peu d'indices archéologiques pour le Mésolithique et le Néolithique ancien (Hamon, 1995 ; Verjux, 1999). Le site de La Tuille, à Nohant-en-Graçay (vallée du Fouzon, Cher), atteste une occupation du Néolithique ancien du V" millénaire av. J.-C., (Deloze, Gauthier, 1996 ; Hamon, 1995). Cette occupation correspondrait au site le plus méridional du groupe de Villeneuve-Saint-Germain (phase finale). En ce qui concerne le Néolithique moven I (première moitié du $V^{c}$ millénaire), il est représenté en région Centre par quelques sites comme Brion, Indre (Hamon, 1995), Nohant-en-Graçay, Cher (Hamon, 1995), Chambon, Indreet-Loire (Hamon et al., 1997), Châtre-sur-Cher, Loir-et-Cher (Hamon et al., 1997) et Saumeray, Eure-et-Loir (Hamon et al., 1997). Les données sont en revanche plus nombreuses à partir du Néolithique moyen, représenté par le groupe de Chambon, contemporain et culturellement proche de la culture de Cerny (Hamon et al., 1997 ; Irribaria, 1997). La synthèse des datations radiocarbone sur la culture de Cerny permet de situer l'occupation humaine en région Centre entre 4550 et 4150 cal. BC (Constantin et al., 1997 ; Guilaine, 1997).

Le Néolithique récent et final est représenté par quelques fouilles d'enceintes ou d'habitats (Hamon, 1997, 1998 ; Villes, 2003). Mais ce qui caractérise cette période, c'est l'exploitation intensive des dalles de silex du GrandPressigny pour la production de grandes lames (fig. 1 et 2 ; Mallet, 1992). Les lames de silex produites en grande quantité ont été exportées dans toute la moitié nord de l'Europe (France, Suisse, Belgique, Luxembourg, Allemagne et Pays-Bas), constituant ainsi ce qu'on peut appeler le " phénomène Grand-Pressigny " (Mallet et al.,
2000, 2004 ; Delcourt-Vlaeminck, 1998, 1999, 2004). En région Centre, les habitats correspondant à ces exploitations intensives de silex n'ont pas été retrouvés, sauf dans quelques cas tels que Les Marais à Barrou (Indre-ct-Loirc), Les Vaux à Moulins-sur-Céphons (Indre) ou Le Petit Paulmy à Abilly (Indre-et-Loire) (Krausz, Constantin, 1995 ; Marquet, Millet-Richard, 2000 ; Villes, 2003). Les caractéristiques géomorphologiques et la nature des constructions (matériaux périssables) pourraient expliquer une mauvaise conservation de ces occupations, mais la rareté des sites d'habitat identifiés ne peut être un argument suffisant pour discuter de l'apparente faiblesse des occupations néolithiques de cette région. La très grande rareté des vestiges provenant de sites funéraires ou d'habitats de cette période, en regard de ccttc cxploitation massive du silex, pose ainsi une des principales interrogations sur le Néolithique final de la région Centre (Airvaux, Primault, 2002 ; Villes, 2003). Quelle était la fonction de ces lames? Quelle est la raison d'une telle production ? Quelle était la place d'une telle production dans le contexte socio-économique du Néolithique final ? Les études tracéologiques ont montré que ces grandes lames en silex ont servi assez souvent à des activités de boucherie, mais l'utilisation la plus systématique a surtout concerné la coupe des végétaux non ligneux tendres de type céréales ou roseaux (Anderson et al., 1992 ; Beugnier, Plisson, 2004 ; Plisson et al., 2002). Pour l'instant, toutes les études tracéologiques ont été réalisées sur des lames retrouvées en Suisse ou dans l'est de la France, c'està-dire dans les régions importatrices. Des analyses tracéologiques sont en cours sur des lames provenant de la région Centre ; elles devraient permettre de savoir si ces lames ont principalement servi à la coupe de végétaux non ligneux tendres. Il n'y aurait alors plus qu'un pas à faire pour émettre l'hypothèse d'une utilisation agricole de ces lames (Beugnier, Plisson, 2004 ; Plisson et al., 2002) et pour relier l'ampleur de cette production et l'intensité de ces exportations à une extension des espaces agraires.

Le Bronze ancien et moyen (2300-1350 BC) est reconnu en région Centre essentiellement par des découvertes dites isolées d'objets en bronze ou de dépôts, faites au cours des labours ou de dragages des rivières. Il s'agit en majorité de trouvailles anciennes et fortuites, sans localisation ni circonstances de découverte précises (Gabillot, 1997). Hormis quelques rares cas tels que Les Marais à Barrou (Indre-et-Loire), les sites d'habitat sont très peu nombreux et ne sont également identifiés que par des traces d'occupation fugaces (fosses avec quelques tessons de poteries 


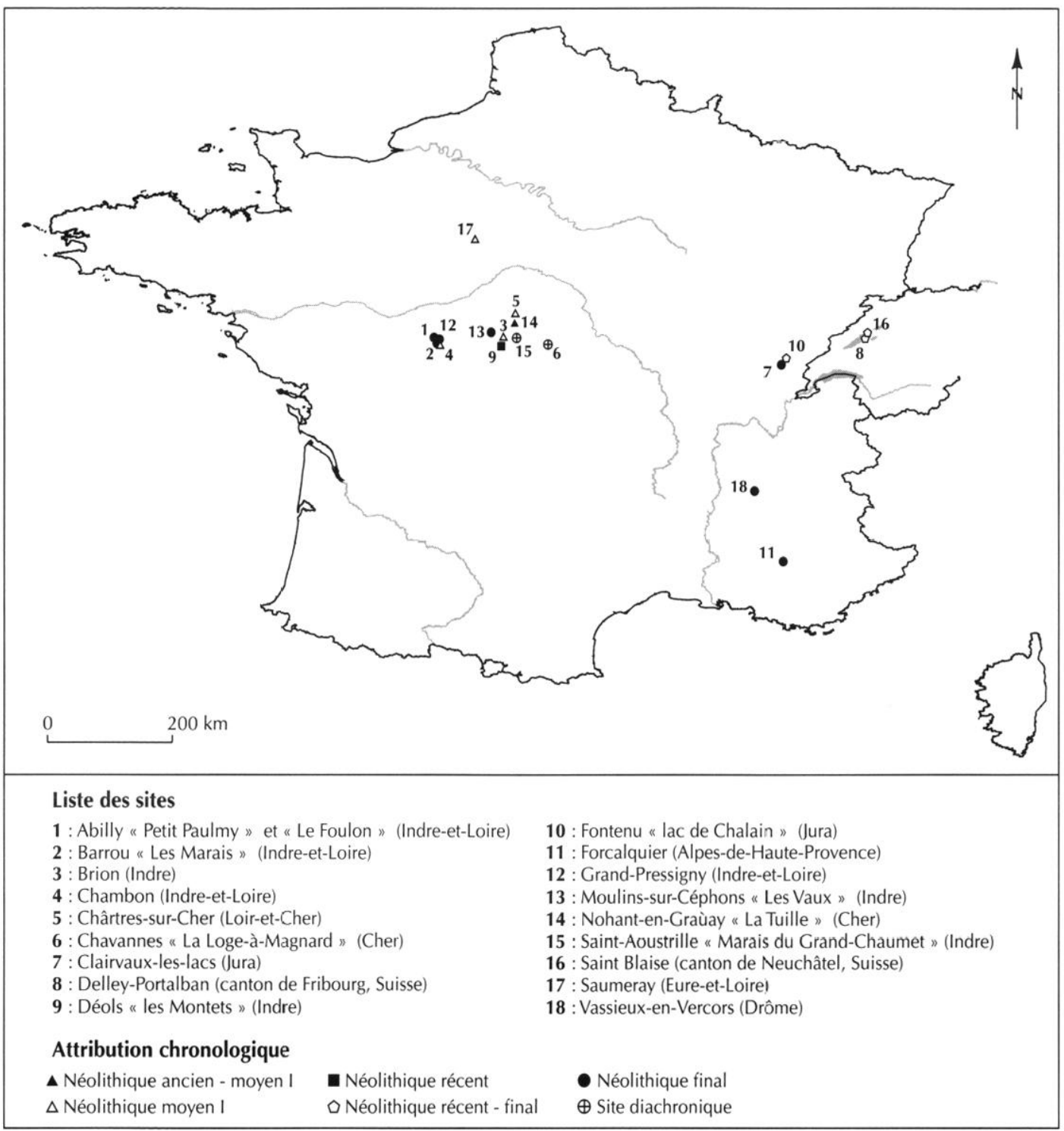

Fig. 2 - Carte de localisation des sites archéologiques cités dans le texte et classés par grandes périodes chronologiques; la déformation est due au système de représentation en projection cylindrique Lambert directe (DAO M. Landré, PGC, MSH Dijon).

caractéristiques, rares trous de poteaux). Les pratiques funéraires sont encore plus mal connues, puisque seule une tombe fouillée anciennement est nettement attribuée à cette période (Gabillot, 1997). Les objets retrouvés témoignent toutefois d'une activité humaine dynamique, dont les productions métalliques semblent synthétiser des courants d'influences nombreux et variés (Gabillot, 1997).

En revanche, à partir du Bronze final et surtout au tout début de l'âge du Fer, l'occupation du sol devient beaucoup plus importante, en premier lieu sous la forme d'habitats de hauteur au Bronze final évoluant ensuite vers un tissu assez dense de fermes dispersées (Buchsenschutz, 1994 ; Augier et al., 2001). De façon générale, l'âge du Fer est considéré comme une période d'importantes mutations qui concernent l'organisation de l'habitat et de l'espace agraire (Buchsenschutz, 1997 ; Chouquer, 2000). Cette évolution démographique et sociale est-elle perceptible dans la transformation du milieu et dans les pratiques agraires ? 


\section{ÉTUDE PALÉOENVIRONNEMENTALE DE LA TOURBIÈRE DE LA LOGE-À-MAGNARD}

\section{SITE, PRÉLÈVEMENTS ET CHRONOLOGIE}

La tourbière de La Loge-à-Magnard (LAM ; fig. 3) se trouve au sud-est de Châteauneuf-sur-Cher sur la commune de Chavannes (Cher). Elle prend place au fond du petit vallon du ruisseau de Chevrier, en bordure de la vallée du Cher, dans les formations marno-calcaires lacustres tertiaires du bassin d'Uzay-le-Vénon (Lablanche, Deprez, 1984). L'accumulation organo-calcaire atteint plus de $3 \mathrm{~m}$ d'épaisseur.

Le prélèvement sur le terrain a été réalisé sous forme de carottage par forage mécanique à l'aide d'une sonde russe de type GYK. Cette sonde ne compacte pas les sédiments, ce qui permet de conserver intact le développement stratigraphique.

La chronologie des dépôts est basée sur trois datations radiocarbone AMS (tabl. I). Après calibration des dates BP en âge calibré (cal. ; Stuiver et al., 1998), un modèle Âge/Profondeur permet d'obtenir une estimation de l'âge des formations sédimentaires à toutes les profondeurs (fig. 4). Dans le texte, les datations radiocarbone calibrées sont mentionnées entre crochets et en années réelles $\mathrm{BC}$ (Before Christ) ; les âges estimés par le modèle Âge/Profondeur sont mentionnés sans crochet.

\section{MÉTHODES D'ANALYSES}

L'étude sédimentologique repose sur des analyses pétrographiques et micromorphologiques (Fédoroff et al., 1989 ; Courty et al., 1989) de 27 lames minces de la séquence sédimentaire continue, non perturbée. Elle vise à reconstruire les conditions de sédimentation qui reflètent les conditions hydrologiques générales (Magny, 1992, 1998) et les périodes d'apport détritique. Les faciès les plus riches en matières organiques, de type tourbes et gyttja, traduisent des périodes de ralentissement des flux hydriques en relation avec des conditions climatiques plus sèches (fig. 5b). Inversement, les faciès les plus carbonatés se mettent en place au moment d'une reprise des écoulements dans la tourbière entrainant une augmentation du niveau d'eau et peuvent être associés à une évolution des conditions climatiques vers une ambiance plus humide. Les contextes de sédimentation étudiés ici sont largement dominés par des dépôts autochtones (matière organique et carbonate), c'est-à-dire produits sur place par accumulation de végétaux ou par précipitation ; la présence localisée d'apport sédimentaire allochtone traduit une érosion des sols et des formations loessiques sur le bassin versant (fig. 3). Ces phénomènes d'érosion accompagnent généralement les phases d'ouverture du milieu, exposant ainsi les sols aux agents atmosphériques et plus particulièrement aux précipitations. Les détails de l'analyse sont décrits dans Vannière (2001) ; seules des reconstructions synthétiques sont présentées ici.

L'analyse du signal incendie a été réalisée par un comptage des particules de charbon de bois préservées dans les sédiments. Ce sont des particules de taille microscopique (10 à $250 \mu \mathrm{m}$ ) dispersées lors des feux, par voie aérienne ou aquatique. Ces particules vont se déposer dans une multitude de zones d'accumulation sédimentairc ; chaque dépôt atteste alors l'occurrence d'un événement lié au feu. L'identification, la caractérisation et la quantification de ces particules permettent de définir le signal incendie (Vannière, 2001). Les observations sont effectuées, en lumière réfléchie, sur des lames de type palynologique (l'observation se fait sur un résidu de traitement chimique servant à l'extraction des pollens et charbons de bois) tous les $2 \mathrm{~cm}$, et sur lames minces (les sédiments sont conservés intacts, sans traitement chimique et sans modification de la structure des dépôts) en continu sur toute la séquence. La présence de particules de taillc supćrieure à $100 \mu \mathrm{m}$ est enregistrée. L'utilisation de la microscopie à réflexion permet de faire la différence entre un matériel organique oxydé ou des minéraux opaques, et les particules carbonisées (fig. 5 ; Vannière, 2001). L'influx en microcharbons de bois ou Charcoal index $\left(\mathrm{mm}^{2} \cdot \mathrm{cm}^{-2} \cdot \mathrm{an}^{-1}\right.$; Clark, 1988) est calculé sur la base des concentrations absolues (Cour, 1974) et du modèle Âge/Profondeur (fig. 4). Cette unité de mesure du signal incendie renvoie à une quantité de microcharbons de bois déposés par année écoulée et permet des

Tabl. I - Datations radiocarbone obtenues sur la séquence sédimentaire de la lourbière de La Loge-à-Magnard (LAM). Les dates radiocarbone ont été calibrées avec le logiciel CALIB 4.3 (Stuiver et al., 1998). Elles sont présentées en âge radiocarbone $(B P)$ et en âges calibrés exprimés en années réelles (cal. $B C$ à $2 \sigma$ ).

\begin{tabular}{|c|l|c|c|c|}
\hline $\begin{array}{c}\mathbf{N}^{\circ} \\
\text { d'échantillon }\end{array}$ & Code labo & $\begin{array}{c}\text { Matériel } \\
\text { analysé }\end{array}$ & Datation BP & Âge calibré \\
\hline CHA-150 & Utc 8936 & tourbe & $3609 \pm 37$ & {$[2117-1829]$ cal. BC } \\
\hline CHA-220 & Utc 8937 & tourbe & $4293 \pm 37$ & {$[3005-2877]$ cal. BC } \\
\hline CHA-325 & Utc 8838 & tourbe & $6461 \pm 36$ & {$[5479-5323]$ cal. BC } \\
\hline
\end{tabular}



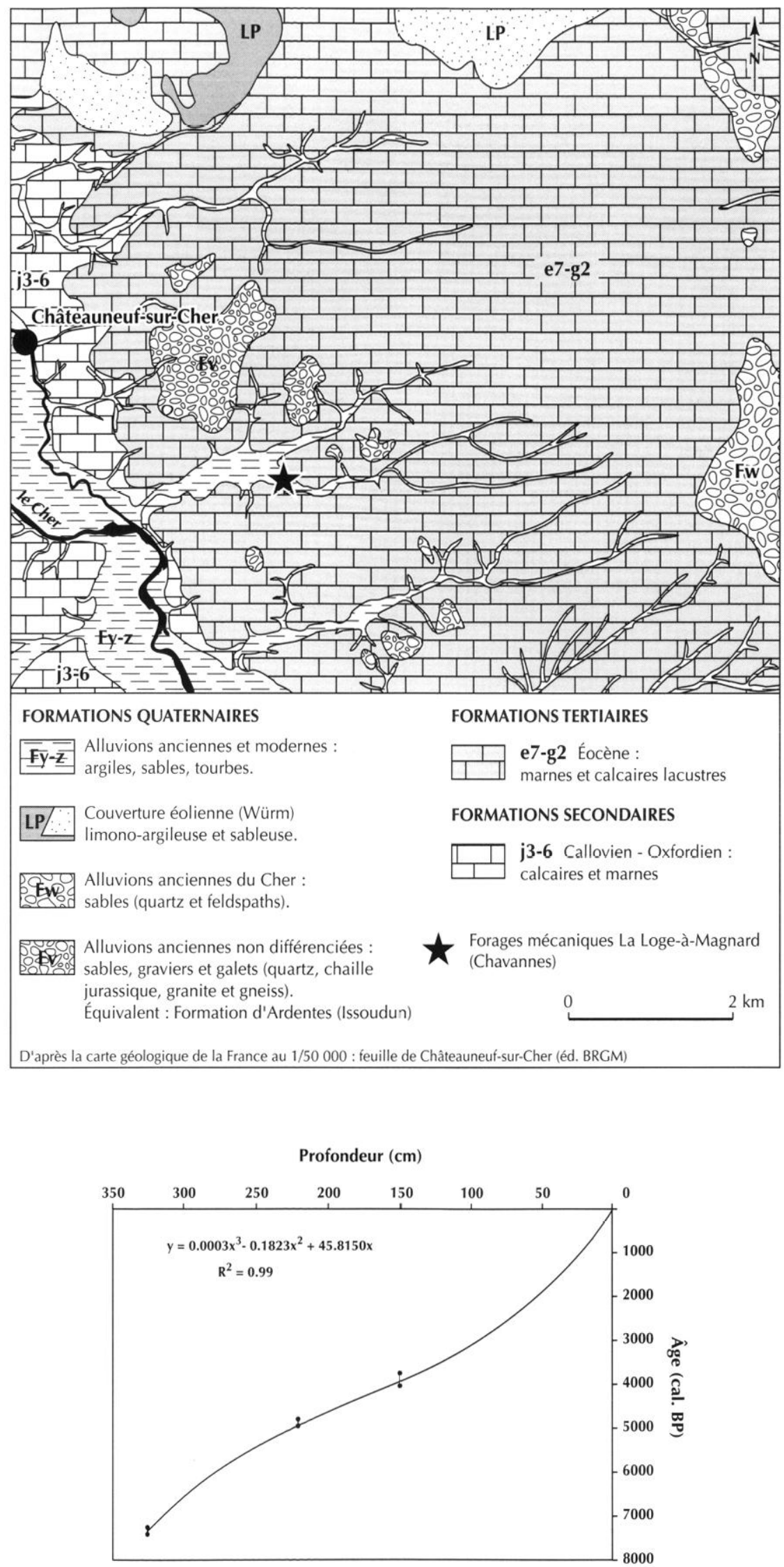

Fig. 3 - Carte géologique simplifiée de la région de Châteauneuf-sur-Cher et localisation de la tourbière de La Loge-à-Magnard (Chavannes, Cher) d'après Lablanche, Deprez, 1984.
Fig. 4 - Modèle Âge/profondeur réalisé à partir des datations radiocarbone cal. $B C \grave{a}$ $2 \sigma$ (tabl. I) obtenues sur la séquence de La Loge-à-Magnard (Chavannes, Cher). 
Fig. 5 - Exemples d'observations microscopiques sur lame mince en lumière transmise (LT) et lumière réfléchie (LR) : $a$, particules organiques opaques dans une matrice carbonatée (LT); ces particules sont pour partie carbonisées et pour partie oxydées; l'accumulation de carbonates dans ce type de contexte atteste un contexte plutôt lacustre; $\boldsymbol{b}$, faciès tourbeux correspondant à un amas de matières organiques amorphes rougeâtres témoin de l'accumulation de végétaux en milieu humide de type marais (LT) ; c, particules organiques carbonisées, opaques en $L T ; d$, microcharbons de bois; ils sont réfléchissant en LR ce qui permet entre autres de déterminer que ce sont des particules issues d'un processus de carbonisation.

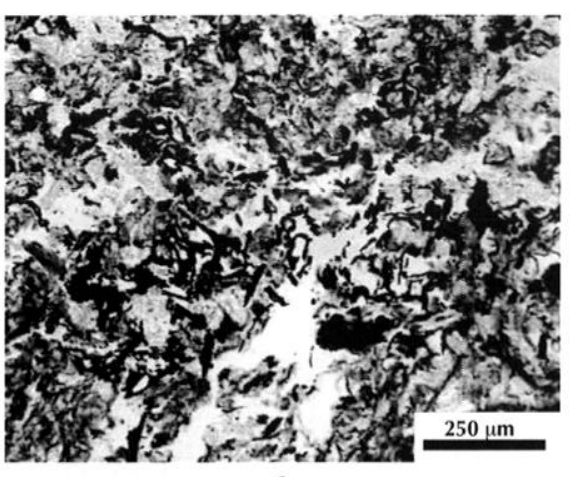

a

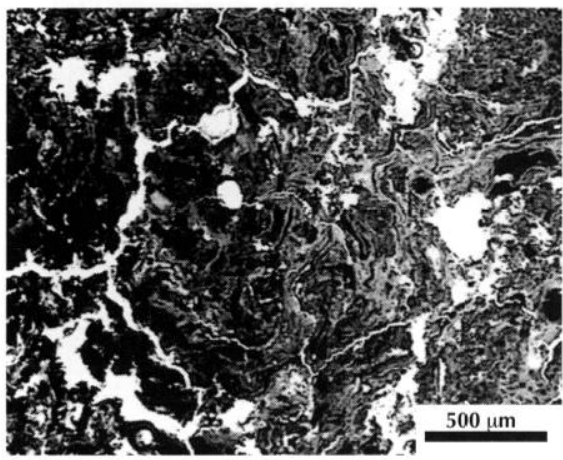

b

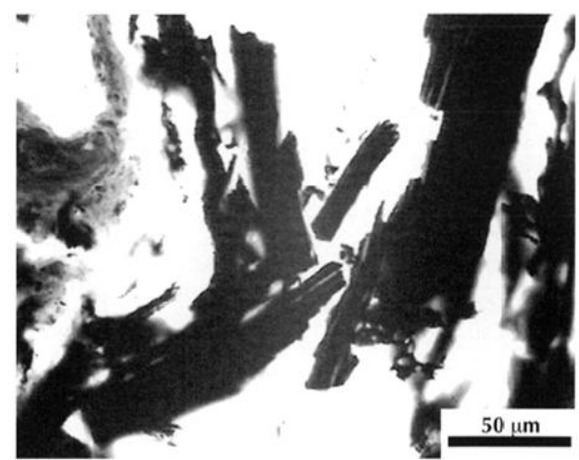

C

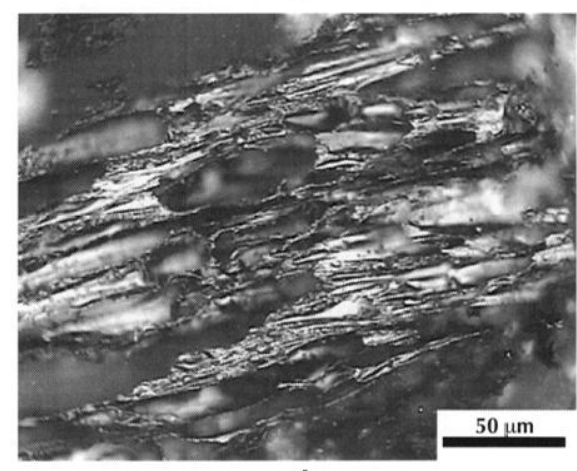

d comparaisons quantitatives entre les différentes périodes considérées. L'amplitude des variations de cet influx en particules carbonisées reflète d'une part la fréquence des occurrences de feux et d'autre part l'ampleur des événements. La présence de particules plus grosses, de taille supérieure à $100 \mu \mathrm{m}$, marque l'occurrence de feux au niveau local et/ou microrégional de grande ampleur (Clark et al. 1998 ; Vannière, 2001), alors que l'absence de ce type de particules tend plutôt à refléter un signal régional et/ou concernant des feux de plus faible ampleur.

\section{RÉSULTATS}

Les trois datations radiocarbone placent chronologiquement cette séquence sédimentaire entre [5179-5323] cal. BC et 700 cal. BC environ (tabl. I ; fig. 4). Sept phases permettent de décrire les évolutions environnementales observées sur près de 5000 ans sur le site de la Loge-àMagnard (LAM), phases 1 à 7 (LAM 1 à LAM 7 ; fig. 6).

Entre [5479-5323] et 3900 cal. BC, les dépôts tourbeux enregistrent successivement trois épisodes de recrudescence des incendies (LAM 1). La présence de particules carbonisées supérieures à $100 \mu \mathrm{m}$ tend à indiquer un signal incendie local, c'est-à-dire des feux ayant eu lieu sur le bassin versant de la tourbière. La sédimentation est relati- vement homogène, les quelques variations marquées par une augmentation de la teneur des sédiments en fraction carbonatée témoignent d'un abaissement du $\mathrm{pH}$ favorisant la précipitation des carbonates, reflet d'une modification des conditions écologiques de la tourbière. Ces modifications sont directement corrélées avec les périodes de chute de l'influx sédimentaire en microcharbons de bois, indices d'une absence quasi totale des incendies. Entre 3900 et 3200 cal. BC, le signal incendie est quasiment nul (LAM 2). Les sédiments deviennent beaucoup plus carbonatés : ils traduisent une remontée du niveau d'eau dans la tourbière et des conditions climatiques plus humides. Vers 3500 cal. BC, la sédimentation à nouveau plus organique marque une nouvelle baisse du niveau des eaux ou du moins un ralentissement des écoulements favorisant le développement de la tourbière, témoin d'une évolution vers des conditions climatiques moins humides. Cet épisode organique s'interrompt vers 3000 cal. BC, moment où un changement majeur s'opère, à la fois dans la nature des dépôts sédimentaires et dans l'occurrence du signal incendie (LAM 3). Une importante fraction détritique vient se déposer dans la tourbière au fur et à mesure que la composante carbonatée des sédiments devient progressivement dominante. Cette fraction détritique se caractérise par des grains de quartz, et plus particulièrement par des fragments de sol et de substrat 


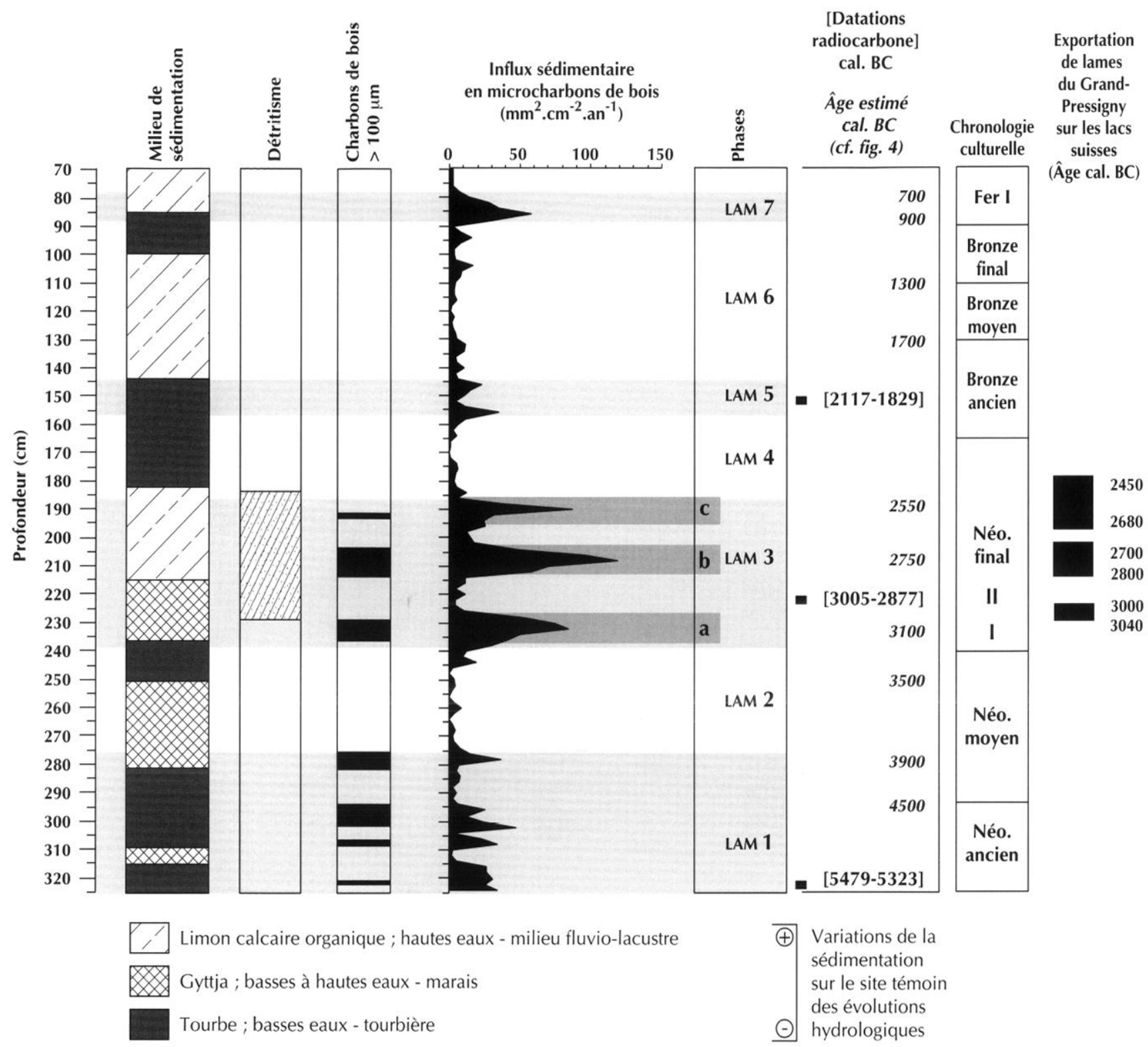

Fig. 6 - Reconstructions paléohydrologiques, signal incendie et chronostratigraphie de la séquence sédimentaire de la tourbière de La Loge-à-Magnard (Chavannes, Cher). Trois pics dans l'influx sédimentaire en microcharbons de bois se distinguent particulièrement vers 3100, 2750, et $2550 \mathrm{cal}$. BC; ils apparaissent contemporains des phases d'exportation des grandes lames en silex du Grand-Pressigny (Indre-et-Loire), utilisées principalement pour la coupe de végétaux non ligneux tendres de type herbacées ou graminées (Mallet, 1992 ; Anderson et al., 1992 ; Plisson et al., 1992).

calcaire ; elle témoigne d'une période de forte déstabilisation et d'une importante érosion des sols sur le bassin versant. Durant cette période, datée autour de [3005-2877] cal. BC une forte recrudescence des feux est également enregistrée. Successivement, vers 3100,2750 et $2550 \mathrm{cal}$. BC, trois importants pics dans l'influx sédimentaire en microcharbons de bois (LAM 3a, 3b et 3c) marquent des occurrences de feux de grande ampleur, bien distinctes dans le temps. Chacun de ces trois pics est confirmé par la présence de particules supérieures à $100 \mu \mathrm{m}$. La phase LAM 4 correspond à un abaissement du niveau d'eau et/ou un ralentissement des écoulements, et à la colonisation du milieu par la végétation hygrophile ; mais également à un arrêt des apports détritiques et de l'érosion sur le bassin versant. Le signal incendie très faible indique une baisse importante de la fréquence des feux. Vers [2117-1829] cal. BC, une légère 


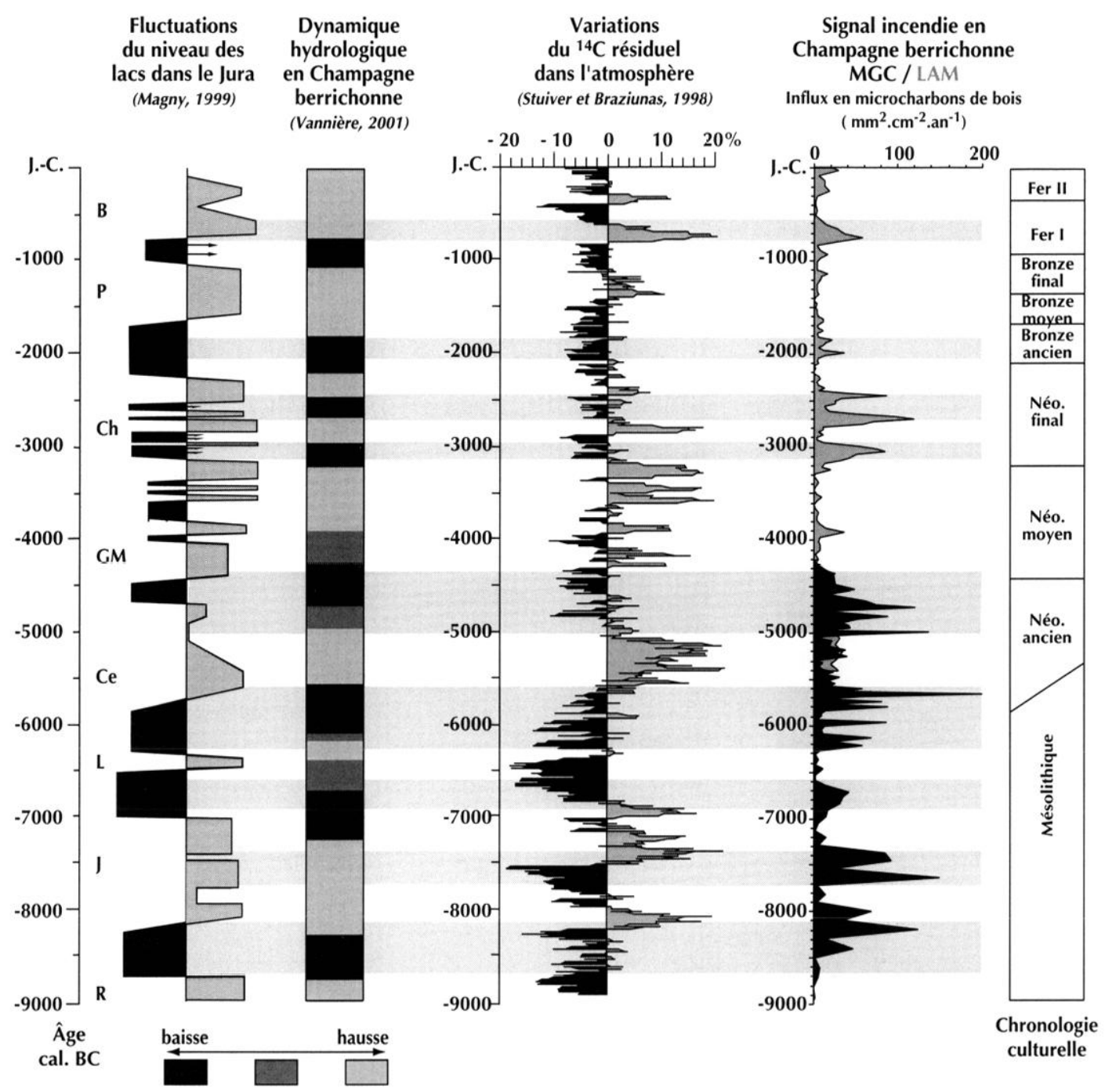

Fig. 7 - Comparaison pour les 9 derniers millénaires av. J.-C. entre les fluctuations du niveau des lacs dans le Jura (d'après Magny, 1999), les variations paléohydrologiques et l'histoire des feux en Champagne berrichonne (Vannière, 2001 ; Vannière, Laggoun-Defarge, 2002) et les fluctuations $d u{ }^{14} \mathrm{C}$ résiduel dans l'atmosphère (Stuiver et al., 1998). Les fluctuations du ${ }^{14} \mathrm{C}$ résiduel dans l'atmosphère peuvent être considérées comme un reflet empirique de l'histoire du climat à l'échelle globale depuis les 12 derniers millénaires. Cette comparaison montre que, jusqu'au Bronze final, la fréquence des feux est corrélée avec les évolutions du climat, attestant ainsi l'importance de ce paramètre dans leur déclenchement. Les reconstructions sur l'histoire des feux proviennent de deux séquences sédimentaires : MGC-Marais du Grand-Chaumet (Saint-Aoustrille, Indre) et IAM - tourbière de La Loge-à-Magnard (Chavannes, Cher).

recrudescence des feux est enregistrée par deux petits pics (LAM 5). Cette occurrence des feux au Bronze ancien s'estompe ensuite progressivement pour atteindre un minimum entre 1700 et 1300 cal. BC (LAM 6). La sédimentation marque alors un retour à un écoulement plus dynamique dans la tourbière, attestant une dégradation des conditions climatiques, sans indice d'une érosion des sols sur le bassin versant. À la fin de la phase LAM 6, le signal incendie augmente alors très légèrement ; les évolutions paléohydrologiques entraînent un abaissement du niveau d'eau sur le site et une nouvelle phase d'accumulation sédimentaire presque exclusivement organique. Cette courte période d'abaissement du plan d'eau laisse ensuite place, aux alentours de 900 cal. BC, à une nouvelle reprise des flux hydriques dans la tourbière (LAM 7). Le signal incendie enregistre alors un épisode (LAM 7) de récurrence des feux, estimé vers 900-700 cal. $\mathrm{BC}$. Les trois dernières phases d'occurrence des feux (LAM 5, fin de LAM 6 et LAM 7) 
présentent un signal incendie d'ampleur beaucoup plus faible que durant les phases LAM 3a, 3b et 3c. De plus, ces épisodes de feu ne sont attestés que par le dépôt de particules de charbon de bois inférieures à $100 \mu \mathrm{m}$, ce qui témoigne d'un signal à l'échelle régionale.

\section{DISCUSSION}

\section{NÉOLITHIQUE ANCIEN ET MOYEN : INCENDIES CLIMATIQUES ET FEUX ANTHROPIQUES}

La reconstruction conjointe des évolutions paléohydrologiques et de l'histoire des feux sur la tourbière de La Logeà-Magnard montre qu'avant 4000-3500 cal. BC (LAM 1 et 2), soit pour la fin du Néolithique ancien et le Néolithique moyen I, l'occurrence des feux s'accorde avec un contexte climatique plutôt favorable à leur déclenchement. En revanche, à partir du Néolithique final et au cours de la Protohistoire, la recrudescence des feux n'apparaît pas systématiquement synchrone des indices d'assèchement des conditions environnementales (fig. 6).

Entre 5000 et 4300 cal. BC, une amélioration du climat est également enregistrée par la baisse du niveau des lacs dans le Jura (fig. 7 ; Magny, 1999). Une baisse des précipitations, une augmentation de l'évapotranspiration et de nombreux incendies sont également observés en Allemagne aux alentours de $5200 \mathrm{cal}$. BC (Clark et al., 1989). Durant la première moitié de l'Holocène (entre 9000 et $4000 \mathrm{cal}$. BC), des corrélations ont pu être établies en Champagne berrichonne entre les périodes de forte fréquence de feux et des périodes marquées par des conditions climatiques plus sèches (fig. 7 ; Vannière, Laggoun-Defarge, 2002). Au cours de cette période, en Grande-Bretagne, de nombreux enregistrements sédimentaires montrent un synchronisme dans les fortes fréquences de feux entre différentes régions, une très bonne corrélation des épisodes de feu avec les fluctuations climatiques, mais aucun indice de l'action de l'homme (Macklin et al., 2000). Ce constat suggère plutôt une influence du climat sur l'intensité et la fréquence des incendies. En Amérique, Millspaugh et al. (2000) et Mohr et al. (2000) montrent que les fortes récurrences de feux du début de l'Holocène correspondent aux maxima d'insolation estivaux de l'hémisphère Nord ; ces maxima se traduisent par une saison estivale sèche et prolongée. En Europe, au début de l'Holocène, la forêt tempérée sous un climat à fort contraste saisonnier avec une saison sèche bien marquée a pu être sensible aux départs répétés de feux et à leur extension sur de grandes surfaces (Bradshaw et al., 1997 ; Vannière, 2001). Ceci tend plutôt à soutenir l'hypothèse d'une origine climatique des feux pour la première partie de l'Holocène. Quelques sites du Néolithique ancien et moyen témoignent d'une occupation humaine durant cette période en région Centre ; néanmoins ils restent des témoignages assez ténus pour expliquer les importantes occurrences de feu à cette époque.

Entre 3900 et 3500 cal. BC (LAM 2), les conditions climatiques plus humides enregistrées dans la tourbière de La Loge-à-Magnard s'accordent avec la phase transgressive de Grand-Maclu dans les lacs du Jura (Magny, 1999) ; aucun feu n'est détecté pendant cette période.

Durant le début de l'holocène, facteurs climatiques et anthropiques permettent donc d'expliquer l'occurrence des incendies. Bien qu'il soit difficile d'en faire la part, il faut cependant considérer que les conditions climatiques ont pu favoriser l'occurrence de feux créant une ouverture du milieu dont l'homme a pu bénéficier. L'homme a également pu profiter des conditions climatiques pour conquérir de nouveaux territoires grâce au feu. Ces constatations sont en accord, tant du point de vue chronologique que fonctionnel, avec les conclusions de Richard et Ruffaldi (1996) et Magny (1992) sur les rythmes d'anthropisation dans le Jura, en relation avec une forte variabilité climatique enregistrée notamment sur les lacs de Chalain et de Clairvaux. Une des hypothèses serait qu'au début du Néolithique, la conquête de l'espace aurait été favorisée à certains moments par l'existence de conditions climatiques favorables. L'homme a certainement utilisé le feu comme un outil de déforestation et d'aménagement des espaces agricoles (Galop et al., 2002), mais ces défrichements ne pouvaient être que d'ampleur modeste, en rapport avec les besoins des populations.

\section{APPROPRIATION DE L'ESPACE NATUREL AU NÉOLITHIQUE FINAL}

Sur la séquence de La Loge-à-Magnard, à la différence des premiers épisodes d'incendie enregistrés avant 4000 cal. BC, au cours du Néolithique final (LAM 3) les très fortes occurrences de feux correspondent à une érosion importante des versants et à des conditions environnementales plutôt humides (fig. 6). Cette évolution diverge des reconstructions climatiques régionales proposées pour la Champagne berrichonne (fig. 7 ; Vannière, 2001) ou le Jura (Magny, 1999). D'une part, les événements de feux ne 
génèrent naturellement que des phénomènes d'érosion limités, d'autre part deux périodes d'amélioration climatique sont enregistrées vers $3200-3000$ cal. BC et 2800-2500 cal. BC. Il faut alors envisager une autre interprétation environnementale de l'enregistrement sédimentaire. Une forte déstabilisation du versant peut être à l'origine d'une modification importante de la nature des dépôts sédimentaires et d'un enrichissement en carbonates indépendamment des conditions hydrologiques. L'érosion importante des sols implique une ouverture du milieu et leur mise à nu, notamment dans le cas de mise en culture (Provensal, Morhange, 1994 ; Vannière et al., 2003). Seuls les concrétionnements calcaires qui apparaissent plus particulièrement au moment de la baisse du signal incendie (Vannière, 2001), attesteraient une réactivation des flux hydriques.

Ainsi, le signal incendie pourrait décrire des périodes d'ouverture du milieu par l'homme, marquées par l'occurrence d'incendies de défrichements et des périodes de dégradation climatique qui verraient un ralentissement dans l'expansion des surfaces agricoles (moins de défrichements par le feu). Sur les bords du lac de Chalain (Jura), les différentes phases d'occupations du Néolithique final se corrèlent parfaitement avec l'évolution des conditions climatiques ; les périodes de migration de population correspondent avec les phases plus humides, et à l'inverse, l'expansion démographique régionale entraînant des défrichements intervient pendant les phases d'abaissement du niveau des lacs (Arbogast et al., 1995, 1996). À partir de 4000 cal. $\mathrm{BC}$, les modifications des paramètres de l'orbite terrestre ont entrainé un déplacement du maximum d'insolation de la Terre vers la saison d'automne (Magny, 1995). Les tendances climatiques à long terme montrent également une réduction graduelle de l'insolation terrestre dans l'hémisphère Nord (COHMAP, 1988). Les premiers impacts régionaux des activités humaines sur la végétation et les sols, dans la vallée du Cher et sur le plateau, sont ćgalcmcnt datés de cette période (Vannière, 2001). Le régime des feux ne dépendrait alors plus seulement des conditions climatiques, mais du développement des sociétés humaines. Sur la séquence de La Loge-à-Magnard, aux incertitudes chronologiques près, les phases d'augmentation et de baisse de la fréquence des incendies oscilleraient entre 300 et 150 ans.

Le premier pic d'incendie du Néolithique final (LAM 3a ; fig. 6), vers 3100 cal. BC correspond chronologiquement au développement du groupe du Montet, stylistiquement proche de la culture Horgen et attesté sur le site du Montet, à Déols, dans l'Indre (fig. 2 ; Hamon, 1997, 1998). Le premier indice d'exportation de lame en silex du Grand-Pressigny est attesté en France orientale par une lame emmanchée retrouvée sur le site de Chalain (station 4, phase 2), datée de 3040 av. J.-C., (Pétrequin, 1994). À cette période, les exportations de lames du Grand-Pressigny sont encore très rares. Avant $3100 \mathrm{cal}$. $\mathrm{BC}$, les lames retrouvées en Suisse occidentale proviennent de Forcalquier (Alpes-deHaute-Provence) et les lames de Suisse orientale sont importées depuis l'Italie du Nord (Monti Lessini) (Barfield, 1987 ; Honegger 2001, 2002).

Entre 3000 et 2800 cal. BC, l'influx en microcharbons de bois montre une très forte réduction des feux. Pendant cette période, une diminution dans le rythme des importations de lames du Grand-Pressigny est également mise en évidence, aussi bien sur les sites suisses que français. Entre 2800 et 2700 cal. BC, apparaît un second pic d'incendie, très marqué (LAM 3b). Cette période correspond exactement au moment du développement de la production de lames issues de la technique "livre de beurre " et à la diffusion massive de ces productions de silex du Grand-Pressigny (Mallet, 1992). Une nouvelle diminution du nombre des défrichements par le feu intervient entre 2700 et 2600 cal. BC ; puis un troisième pic d'incendie est enregistré entre 2600 et 2500 cal. BC (LAM 3c). Or, après une diminution importante des exportations de lames vers $2700 \mathrm{cal}$. $\mathrm{BC}$, un fort développement de celles-ci est à noureau observé entre 2680 et 2450 cal. BC. D'après N. Mallet (1992), cette période pourrait correspondre à l'exploitation la plus intensive des silex turoniens du Grand-Pressigny, destinés à l'exportation. Ces données sont parfaitement confirmées par les résultats de Honegger (2001) sur les sites de Saint-Blaise et Delley-Portalban (Suisse). La même tendance chronologique a également été observée aux PaysBas, où la majorité des importations pressigniennes est comprise entre 2600 et 2450 cal. BC (Van der Waals, 1991).

À partir de 2450 cal. BC., les sites archéologiques ne livrent presque plus de grandes lames, mais surtout des pièces de réemploi, de plus en plus nombreuses. Ceci témoigne d'une baisse de la production et des échanges à partir de cette date (Mallet, 1992). Les exportations de silex du Grand-Pressigny cessent totalement à partir de 2450-2400 cal. BC. Il faut alors attendre plus de 500 ans ([2117-1829] cal. $\mathrm{BC}$ ) pour observer de nouvelles occurrences significatives de feux. À l'échelle régionale, la comparaison entre l'histoire des feux et les variations chronologiques d'exportation de lames de silex du Grand-Pressigny, entre 3100 
et 2500 cal. BC, montre une adéquation entre les périodes d'intensification des productions et des exportations de lames de silex et les fortes recrudescences des féux. Au-delà des incertitudes chronologiques, la nature, le nombre et la succession des événements mis en évidence valident la concordance des résultats.

91,4\% des outils taillés dans la région du GrandPressigny, puis exportés, sont des lames (Mallet, 1992). Il est alors envisageable de proposer un lien entre le phénomène de production intensive et de diffusion massive des lames en silex du Grand-Pressigny, destinées à la coupe de végétaux non ligneux de type graminée et herbacée, et les feux d'origine anthropique révélant des défrichements importants. Ce lien attesterait également l'utilisation du feu à grande échelle dans les pratiques agraires et un système de culture sur abattis-brûlis. Ces résultats montrent qu'au Néolithique final, la région Centre est occupée par des populations défrichant de façon répétée la forêt, leur permettant ainsi de développer leurs activités agropastorales. Cette expansion territoriale peut être le fruit d'innovations techniques ou fonctionnelles et/ou favoriser ces innovations. Toutefois, à l'origine, ces variations quantitatives des défrichements et des productions d'outils agricoles ne pourraient-elles pas alors refléter les évolutions paléodémographiques?

On postule qu'une augmentation démographique entraîne nécessairement une augmentation des besoins alimentaires, répercutés et retranscrits par un développement de l'agriculture et donc une amplification de l'impact de l'homme sur son environnement. Vers $3100 \mathrm{cal}$. BC, s'agit-il d'une augmentation démographique et/ou d'une expansion territoriale qui aurait entraîné la mise en place d'un important système de production d'outils agricoles? Étant donné qu'à cette période la production et l'exportation intensive de lames du Grand-Pressigny à partir de " livres de beurre " n'ont pas encore commencé, le premier pic de défrichement doit être considéré comme précurseur du phénomène Grand-Pressigny et pourrait témoigner de la mise en place de populations du Néolithique récent (groupe du Montet).

Ensuite, vers 2800 cal. BC, la production massive de lames a pu constituer une réponse technique au développement important de la démographie à cette période, entraînant une forte demande en outils de récolte des graminées/herbacées pour répondre aux besoins alimentaires. Dans tous les cas, ces résultats pourraient renforcer l'hypothèse d'une augmentation démographique au
Néolithique final par rapport au Néolithique moyen. Une telle variation démographique a également été observée par l'étude du nombre de villages identifiés sur les lacs de Chalain et Clairvaux, dans le Jura (Pétrequin dir., 1997). Un certain nombre d'évolutions techniques sont constatées suite à cette évolution du paléopeuplement (Pétrequin et al., 1998), ainsi qu'une accélération de l'érosion liée à des ouvertures durables du milieu par brûlages répétés (Le Jeune, 1997).

Clark et al. (1989) évoquent pour la période comprise entre 3500 et 2500 cal. BC une intensification de l'ouverture des forêts dans le centre de l'Europe. Entre 3400 et 2800 cal. BC, dans les Pyrénées, la récurrence des feux et des IPA témoigne de la mise en culture de l'espace montagnard de hasse et moyenne altitude et de pâturages dans les zones les plus hautes (Vannière et al., 2001 ; Galop et al., 2003).

En France, plusieurs ateliers de production de grandes lames de silex ont déjà été identifiés, à Forcalquier dans les Alpes-de-Haute-Provence (Renault, 1998), à Vassieux-enVercors dans la Drôme (Malenfant et al. 1971), en Charente (Cordier, 1956), Charente-Maritime (Fouéré, 1994) ou encore en Dordogne (Chevillot, 1989). Au Néolithique final, dans tout le Midi de la France, de longues lames de silex, brutes ou retouchées, ont également été utilisées comme faucilles (Guilaine, 1991, p. 51).

La France, les Pays-Bas, la Belgique, l'Allemagne, le Luxembourg et la Suisse sont concernés par la diffusion massive des couteaux sur grandes lames en silex produits dans la région du Grand-Pressigny (Indre-et-Loire). En Europe occidentale, ce phénomène des grandes lames débute vers 3500 avant J.-C. et se développe au Néolithique récent, puis surtout au Néolithique final, jusque vers 2400 cal. BC. Les productions massives de grandes lames dans différents ateliers français et européens, à partir de plusieurs techniques de débitage et de plusieurs matériaux, et leur diffusion dans toute l'Europe, constituent un phénomène très marquant. La contemporanéité de ces productions permet de parler d'un véritable " phénomène de production des grandes lames "à l'échelle européenne, concernant les Pays-Bas, la Belgique, l'Allemagne, le Luxembourg, la Suisse, la France, l'Espagne, le Portugal et l'Italie et auquel appartient le phénomène Grand-Pressigny.

L'ampleur des événements observés en région Centre, concernant les feux et les productions lithiques, ainsi que la récurrence des indices à l'échelle européenne, pourraient attester un phénomène d'anthropisation de grande importance. Cette anthropisation et cette appropriation de 
l'espace se caractériseraient par la mise en place d'un système agraire adapté répondant aux besoins d'une population grandissante, inscrite dans des réseaux d'échange d'outils, de techniques et de pratiques agraires bien développés. Il apparaît alors que le Néolithique final a marqué une rupture dans l'évolution des écosystèmes et que l'agrosystème est alors devenu un espace significatif dans le paysage.

Ainsi, l'hypothèse proposée consiste à penser que défrichements intensifs et production massive d'outils agricoles pourraient témoigner de la nécessité de répondre à une importante augmentation des besoins alimentaires, liée à une croissance démographique. Il s'agit probablement d'un phénomène d'ampleur géographique et chronologique, mais aussi socio-économique, plus large que le seul phénomène de production de lames dans la région du Grand-Pressigny, ce qui nécessitera l'étude et la comparaison de nouvelles séquences paléoenvironnementales dans d'autres régions.

\section{SYSTÈME AGRAIRE FORESTIER ET POST-FORESTIER : DU NÉOLITHIQUE FINAL AU DÉBUT DE L'ÂGE DU FER}

Entre 2500 et 1800 cal. $\mathrm{BC}$, la tourbière de La Loge-àMagnard enregistre une évolution vers des conditions climatiques moins humides (fig. 6), reconnue également par une importante baisse du niveau des lacs dans le Jura (Magny, 1999). Le signal incendie est quasiment nul jusque vers [2117-1829] cal. $\mathrm{BC}$, date à laquelle il montre deux petits pics attestant une légère recrudescence des feux. L'origine de ces feux peut être liée aux occupations humaines de l'âge du Bronze ancien en Champagne berrichonne, même si l'attribution chronologique de ces sites aux différentes périodes de l'âge du Bronze reste encore imprécise (Gabillot, 1997). Vers 2100 cal. BC, Tinner et al. (1999) observent en Suisse une recrudescence importante des feux corrélée avec l'anthropisation du milieu. Entre 1800 et 1000 cal. BC, la hausse du niveau d'eau dans la tourbière de La Loge-à-Magnard marque une augmentation de la pluviométrie, contemporaine d'une augmentation importante et générale du niveau des lacs dans le Jura et sur le Plateau suisse (Magny, 1999 ; fig. 7). Aucun feu n'est alors enregistré sur la tourbière de La Loge-à-Magnard. Or, dans l'Est de la France, le Bronze moyen correspond à une période de mutation importante dans l'habitat et à une déprise agricole importante (Mordant, Richard, 1993 ;
Richard, 1994, 2000). Au cours du Bronze final, une légère augmentation de l'influx en microcharbons de bois peut être le reflet d'une reprise des activités humaines, alors même qu'une baisse du niveau d'eau dans la tourbière indique une amélioration climatique synchrone d'une régression du niveau des lacs dans le Jura vers $1000 \mathrm{cal}$. BC (Magny, 1999). Vers 900-700 cal. BC, soit durant le premier âge du Fer, une forte augmentation du signal incendie est enregistrée sur les sites de La Loge-à-Magnard (fig. 6) et de Vierzon, Cher (Vannière, 2004). Ces feux sont les indices de défrichements importants, au moment où une très forte augmentation du nombre de sites d'habitat est constatée (Milcent, 1999 ; Augier et al., 2001). En montagne jurassienne, comme en basse Mayenne, les données polliniques montrent un fort impact anthropique marqué par une ouverture importante du milieu forestier à partir du début du I ${ }^{\text {er }}$ millénaire av. J.-C. (Barbier, 1999 ; Richard, 2000).

Ainsi, la recrudescence des incendies, qui caractérise la phase LAM 7 (fig. 6), correspondrait à une forte croissance de la population, à la création de nouveaux espaces agricoles et à une nouvelle organisation du territoire (Buchsenschutz, 1994, 1997 ; Chouquer, 2000 ; Augier et al., 2001). Sur le plan paléohydrologique, le tout début de l'âge du Fer se caractérise par une dégradation climatique, marquée dans la tourbière de La Loge-à-Magnard par une remontée du niveau d'eau, également enregistrée dans les lacs alpins et sur de nombreuses tourbières européennes, où le passage de conditions relativement sèches et chaudes à un climat plus humide et plus frais entraîne une remontée générale du niveau des nappes phréatiques (Van Geel et al., 1999).

Les derniers épisodes de recrudescence des feux (LAM 5 et 7 ; fig. 6), du Bronze ancien et final et du premier âge du Fer, sont enregistrés avec une moindre ampleur que pour le Néolithique. De plus ils ne sont représentés que par des particules de charbons de bois inférieures à $100 \mu \mathrm{m}$. Ce signal est lié soit à des feux plus régionaux, soit à des feux de nature, d'ampleur et d'intensité différentes des feux caractéristiques des défrichements néolithiques.

La taille des microcharbons de bois semble en général directement liée à l'ampleur des feux (Clark et al., 1998 ; Vannière, 2001 ; Vannière et al., 2001). Les variations du signal incendie durant l'âge du Bronze et l'âge du Fer peuvent alors être interprétées comme le résultat d'un changement dans l'utilisation du feu. Les feux de défrichement (essartage) du Néolithique deviendraient des feux d'entretien de l'espace agropastoral, qui produisent des particules 
plus petites, en quantité faible. Ces feux sont plus difficiles à mettre en évidence par une analyse du signal incendie, celui-ci étant alors plus continu mais de moins forte intensité (Clark, Royall, 1995). Le signal incendie serait alors essentiellement représenté par des particules déposées suite à l'érosion des sols et donc par des particules plus fragmentées (Vannière et al., 2001). Clark et al. (1989) en Allemagne, Galop et al. (2002) dans les Pyrénées ont également observé une baisse générale du régime des feux, interrompue par quelques augmentations ponctuelles, durant l'âge du Bronze et l'âge du Fer. Ces auteurs l'expliquent par un changement dans le système agraire : le système de culture sur abattis-brûlis serait abandonné. Au cours du Bronze final et au début de l'âge du Fer, en Champagne berrichonne, l'augmentation des apports sédimentaires dans les grandes vallées suggère une modification importante du schéma d'évolution environnementale, due à une dégradation générale des conditions climatiques (augmentation des flux hydriques et de l'érosion) et à une mise en valeur agricole accrue des bassins versants sur la longue durée, limitant la régénération forestière (Vannière, 2001, 2004). Cette transformation correspond à la mise en place d'un système agraire permanent en relation avec une nouvelle organisation du territoire. Quelques sites du premier âge du Fer sont répertoriés dans le secteur : deux enceintes de hauteur, un habitat isolé et deux tumulus du premier âge du Fer (Milcent, 1998 ; Batardy et al., 2001). À cette période se produit une importante mutation vers un système agraire " postforestier ", avec jachère et élevage pastoral associé, répondant probablement à de nouvelles orientations économiques et à une structuration sociale importante. Cette période correspond également au moment où le développement de la métallurgie offre de nouvelles perspectives et où le bois devient peu à peu une ressource économique de plus en plus importante pour la transformation du minerai (Dieudonné-Glad, 1996).

$$
\text { * } *
$$

L'étude sédimentologique de la tourbière de La Loge-àMagnard montre une évolution paléoenvironnementale, entre le Néolithique ancien et le début de l'âge du Fer, marquée par des périodes de fortes occurrences de feux et d'érosion des sols. Durant la première partie de l'Holocène, jusqu'au Néolithique ancien, le régime des feux semble essentiellement contrôlé par les conditions climatiques. Ces évolutions environnementales, et notamment les ouvertures du milieu associées aux feux, ont pu favoriser l'homme, ses activités et son occupation du milieu. À partir du Néolithique moyen et final, les occurrences de feux et les phénomènes d'érosion semblent pour partie d'origine anthropique et correspondent à la mise en place et au développement des systèmes agraires pré- et protohistoriques. Les reconstructions des dynamiques hydrologiques permettent alors de voir comment, du Néolithique à l'âge du Bronze, les périodes d'expansion agraire s'accordent avec les améliorations des conditions climatiques.

En région Centre, les récurrences de feux enregistrés au cours du Néolithique final, au début et à la fin de l'âge du Bronze et au début de l'âge du Fer correspondent à la mise en place des communautés humaines et aux défrichements nécessaires à l'aménagement de l'espace agraire (fig. 6). Les occurrences simultanées de feux de grande ampleur et de productions laminaires importantes en silex du GrandPressigny attestent une modification des pratiques agraires et peut-être une expansion territoriale importante qui pourraient répondre à une augmentation des besoins alimentaires liés aux variations paléodémographiques. Les variations d'intensité de l'influx en charbon de bois et des processus d'érosion entre le Néolithique et l'âge du Fer reflètent également une évolution dans le système agraire. Les sociétés sont progressivement passées d'un système agraire à culture sur abattis-brûlis itinérant à un système agraire post-forestier, avec jachère et élevage pastoral associé.

Au cours de l'âge du Bronze, les périodes de dégradation des conditions climatiques sont synchrones d'une déprise agraire et/ou d'une modification importante des systèmes économiques et sociaux qui vont entraîner une modification durable des systèmes agraires.

L'approche interdisciplinaire, naturaliste et archéologique, des systèmes agraires permet d'étudier les relations entre les sociétés et leur environnement. Les synchronismes entre reconstructions paléoenvironnementales et données archéologiques montrent la signification macrorégionale de l'enregistrement sédimentaire et la valeur des études sur les influx en microcharbons de bois pour une lecture dynamique des processus d'anthropisation. Le modèle proposé pour le Néolithique final consiste à relier dans un cadre systématique des modifications environnementales d'origine anthropique et la production-diffusion d'outils participant aux fonctionnements des systèmes agraires. Ce modèle pourrait également être testé pour d'autres périodes du Néolithique ou des âges des Métaux. 
De plus, les similitudes observées avec d'autres régions françaises et européennes impliquent qu'il s'agit de phénomènes de grande ampleur, inscrits dans une dynamique évolutive générale de longue durée, soumise aux facteurs communs que sont la démographie et le climat. Ainsi, l'étude régionale présentée ici invite à développer de nouvelles études de cas qui permettront des comparaisons et des synthèses à une plus large échelle.

\section{Remerciements}

Cette étude a été financée par le PCR "Occupation du sol dans la cité des Bituriges Cubi "dirigé par O. Buchsenschutz et F. Dumasy (ENS et université de Paris-I ; Fonds FEDER et FSE).
Les auteurs tiennent à remercier G. Bossuet, $\mathrm{O}$. Buchsenschutz, A. Maussion et $\mathrm{H}$. Richard pour leur aide sur le terrain. Les auteurs remercient M. Gabillot pour leur avoir fourni un état de la documentation, non publié, des occupations de l'âge du Bronze en région Centre et M.-A. Rodot pour sa participation à la cartographie des sites archéologiques. Les auteurs remercient également tous ceux qui ont aidé à la réalisation de cet article par leurs nombreuses discussions, et notamment T. Hamon, H. Plisson, C. Verjux et A. Villes. Les auteurs souhaitent enfin remercier C. Heinz, J. Vaquer et un correcteur anonyme pour leurs relectures du manuscrit et leurs commentaires constructifs. 


\section{BIBLIOGRAPHIE}

Airvaux J., Primault J.

2002 : "Considérations sur l'extension géographique du Néolithique final à "livre de beurre" en Touraine et Poitou (France) ", L'Anthropologie, 106, 2, p. 269-294.

Anderson P., Plisson h., Ramseyer D.

1992 : " La moisson au Néolithique final: approche tracéologique d'outils en silex de Montilier et de Portalban ", Archéologie suisse, 15, 2, p. 60-67.

\section{Arbogast R.-M., Magny M., Pétrequin P.}

1995 : " Expansions et déprises agricoles au Néolithique : populations, cultures céréalières et climat dans la Combe d'Ain (Jura. France) de 3700 à 2500 av. J.-C. ", in VAs DER L.FFUw S. (F.D.), L'homme et la dégradation de l'environnement, Actes des $X V^{p}$ rencontres internationales d'archéologie et d'histoire d'Antibes, 20-22 oct. 1994, SophiaAntipolis, éd. APDCA, p. 19-41.

1996 : « Climat, cultures céréalières et densité de population au Néolithique : le cas des lacs du Jura français de 3500 à 2500 av. J.C. ", Archäologisches Korrespondenzblatt. 26,2 , p. 121-144.

Augier L., Milcent P.Y., Buchsenschutz O.

2001 : "Les groupes de l'âge du Fer. De l'âge du Bronze à la cité des Bituriges ", in Batardy C., Buchse.ssche'Tz O., DCMasy F. (F.D.), Le Berry antique - Atlas 2000, Suppl. 21 à la Revue archéologique du Centre de la France, p. 158-159.

\section{BARBIER D.}

1999 : "Histoire de la végétation du nordmayennais de la fin du Weichsélien à l'aube du XXI" siècle. Mise en évidende d'un Tardiglaciaire armoricain ", in Interactions Homme-Milieu, 2, Nantes, Groupe d'études des milieux naturels (GEMINA), $284 \mathrm{p}$.

\section{BARFIELD L. H.}

1987 : « Recent work on sources of Italian flint ", in SiEveking G. G., Nrwcommer M. H. (Éd.), The Human Uses of Flint and Chert, International Flint Symposium 4, Brighton, April $10^{\text {h }}-15^{\text {th }} 1983$, Cambridge University Press, p. 231-239.

Batardy C., Buchsenschutz O., Dumasy F. (ÉD.)

2001 : Le Berry Antique - Atlas 2000, Suppl. 21 à la Revue archéologique du Centre de la France, $192 \mathrm{p}$.
Beugnier V., Puisson H.

2004 : " Les poignards en silex du GrandPressigny : fonction de signe et fonctions d'usage ", in Bod: P., Constantin C. (DIR.), Approches fonctionnelles en Préhistoire, Actes du XXV congrès préhistorique de France, Nanterre, 24-26 nov. 2000, Paris, éd. Société préhistorique française, p. 139-154.

Bradshaw H. W., Tolonen K., Tolonen M. 1997 : "Holocene Records of Fire from the Boreal and Temperate Zones of Europe ", in Clatre J. S., CACHIER H., Goldammer J. G., Stocks B., Sediment Records of Biomass Burning and Gilobal Change, NATO-ASI series I, Global environmental change, 51, New York, Springer Verlag, p. 347-365.

BUCHSENSCHUTZ 0 .

1994 : «Introduction ”, in BLCHSFNSCHUT\% O., MÉNIEL P. (DIR.), Les installations agricoles de l'age du Fer en Île-de-France, Actes du colloque de Paris 1993, Paris, Presses de l'École normale supérieure, (coll. Étude d'histoire et d'archéologie, IV), p. 9-24.

1997 : « Réflexion sur les parcellaires de l'âge du Fer en France septentrionale ", in Blrnolf J., Bravard J.-P., Choululer G. (E.D.), La dynamique des paysages protohistoriques, antiques, médiévaux et modernes, Actes des XVIF rencontres internationales d'archéologie et d'histoire d'Antibes, 19-21 oct. 1996, Sophia-Antipolis, éd. APDCA, p. 13-20.

Buchsenschutz O., Coulon G., Gratier M., Shesse A., Holmgren J., Mills M., Orssaud D., Querrien A., Rialland Y., SoYer C., TABbagh A.

1988: L'évolution du canton de Levroux d'après les prospections et les sondages archéologiques, Suppl. 1 à la Revue archéologique du Centre de la France, Levroux I, $182 \mathrm{p}$.

\section{Carcaillet C}

1998: “A spatially-precise study of Holocene fire history, climate and human impact within the Maurienne valley, North French Alps ", Journal of Ecology, 86, 1, p. 384-396.

\section{Chevillot C.}

1989 : Sites et cultures de l'âge du Bronze en Périgord, Périgueux, Vesuna (Archéologies, 3), 2 vol.

CHOUQuer G.

2000 : L'étude des paysages - Essais sur leurs formes et leur histoire, Paris, éd. Errance, $208 \mathrm{p}$.

\section{Clark J. S.}

1988 : "Stratigraphic charcoal analysis on petrographic thin sections : application to fire history in northwestern Minnesota ", Quaternary Reseach, 30, p. 81-91.

Clark J. S., Lynch J., Stocks B. J., GoldAMmer J. G.

1998 : "Relationships between charcoal particles in air and sediments in westcentral Siberia ", The Holocene, 8, p. 19-29.

\section{Cilark J. S., Merk J., Müller h.}

1989, "Post-Glacial fire, vegetation, and human history of the northern Alpine forelands, south-western Germany ", Journal of Ecology, 77, p. 897-925.

\section{Clark J. S., Royall P. D.}

1995 : "Transformation of a northern hardwood forest by aboriginal (Iroquois) fire: charcoal evidence from Crawford Lake, Ontario, Canada ", The Holocene, 5 , p. 1-9.

\section{COHMAP members}

1988 : "Climatic Changes of the last 18000 years. Observations and Model Simulations", Science, 241, p. 1043-1052.

Constantin C., Mordant D., Simonin D.

1997 : « La culture de Cerny et le Chalcolithique de la terminologie européenne ", in La culture de Cerny. Nouvelle économie, nouvelle société au Néolithique, Actes du colloque international de Nemours, mai 1994, Nemours, èd. APRAIF (Mémoires du musée de Préhistoire d'île-de-France, 6), p. 701-710.

\section{Cordier G.}

1956 : "Un atelier pressignien en Charente: les Martins, communc de Mouthiers ", in Actes du congrès préhistorique de France, $X V$ session, Poitiers/Angoulême, Paris, éd. Société préhistorique française, p. $388-402$.

\section{Cour P.}

1974: "Nouvelles techniques de détection des flux et des retombées polliniques: étude de la sédimentation des pollens et des spores à la surface des sols ", Pollens et Spores, XVI, 1, p. 103-141. 
Courty M.-A., Goldberg P., MACPhaIl R. I. 1989 : Soils and Micromorphology in Archaeology, Cambridge University Press, 344 p.

\section{Delcourt-Vlaeminck M.}

1998: Le silex Grand-Pressigny dans le nord-ouest de l'Europe. Le silex tertiaire, concurrent possible du Crand-Pressigny?, thèse de doctorat, Toulouse, EHESS.

1999 : « Le silex du Grand-Pressigny dans le nord-ouest de l'Europe ", Bulletin de la Société des amis du musée du Cirand-Pressigny, 50, p. 57-68.

2004 : " Les exportations du silex du GrandPressigny et du matériau tertiaire dans le nord-ouest de l'Europe au Néolithique final/Chalcolithique ", in VAN DER LINDEN M., Salanova L. (DIR.), Le troisième millénaire dans le nord de la France et en Belgique, Actes de la journée d'études SKBAP. SPF, 8 mars 2003, Lille, Mémoires de la Société préhistorique française, XXXV, Anthropologica et praehistorica, Bulletin de la société royale belge d'anthropologie et de préhistoire, 115, p. 139-154.

Deloze V., Gauthier A.

1996 : "Évolution de l'environnement sédimentaire et végétal du site archéologique de "La Tuille" " (Vallée du Fouzon, Cher, France), in L'Archéométrie dans les pays européens de langue latine, Actes du colloque GMPCA de Périgueux 26-29 avril 1995, Suppl. à la Revue d'Archéométrie, p. 211-216.

\section{Dieudonné-Glad N.}

1996 : " Exploitation minière et forestière: artisanat du fer et nature dans l'Antiquité chez les Bituriges ", in Colardell.te M. (DIR.), L'Homme et la nature au Moyen Age, Actes du $V^{*}$ congrès international d'archéologie médiévale, Grenoble, Paris, éd. Errance, p. 222-227.

Fedoroff N., Courty M. A., Thompson M. L. 1989 : " Micromorphological evidence of paleoenvironmental change in Pleistocene and Holocene palaeosols ", in Dougias L. A. (ÉD.), Soil Micromorphology : a basic and applied Science, New York, Elsevier Sci., p. 653-665.

\section{FoUÉRÉ P.}

1994 : Les industries en silex entre Néolithique moyen et Campaniforme dans le nord $d u$ Bassin aquitain, thèse de doctorat, univ. de Bordeaux-I.

\section{Gabillot M.}

1997: Le Bronze moyen en région Centre, mém. de maîtrise, univ. de Bourgogne, multigraphié, $70 \mathrm{p}$.
Galop D., Vannière B., Fontugne M.

2002 : " Human activities and fires history since $4500 \mathrm{BC}$ on the Northern slope of the Pyrenees: a record from Cuguron (central Pyrenees) ", Oxford, BAR, International Series, 1063, p. 43-51.

\section{Galop D., Vannière B., Lopez-Saez A.}

2003 : "Des abattis-brûlis néolithiques au système agro-pastoral actuel. Mise en évidence pluridisciplinaire de l'évolution $\mathrm{du}$ système agraire dans une vallée du piémont pyrénéen entre le Néolithique ancien et l'Antiquité ", in Pirineus i veins al $3^{r}$ Millenni AC, Actes du XIr colloque d'archéologie de Puigcerda, Moia, Publicacions de l'Institut d'estudis ceretans, p. 261-274.

\section{GUILAINE J.}

1997 : «Pour conclure: Cerny, Cernoìdes, Chalcolithique ", in La Culture de Cerny. Nouvelle économie, nouvelle société au Néolithique, actes du colloque international de Nemours 1994, Nemours, éd. APRAIF (Mémoires du musée de Préhistoire d'Îlede-France, 6), p. 711-715.

2000 : "Changeons d'échelles: pour la très longue durée, pour de larges espaces " in GuIlaine J. (DIR.), " La très longue durée ", Études rurales, 153-154, p. 9-12.

GuILAINE J. (DIR.)

1991 : Pour une archéologie agraire : à la croisée des sciences de l'homme et de la nature, Paris, Armand Colin.

\section{HAMON T.}

1995 : « Installations du Néolithique ancien et moyen I à Nohant-en-Graçay (Cher) et à Brion (Indre) ", Revue archéologique du Centre de la France, 34, p. 157-176.

1997, "L'enceinte néolithique "du Montet” à Déols (Indre). Note préliminaire ", Bulletin de la Société préhistorique française, 94, 2, p. 230-236.

1998 : “L'enceinte néolithique du Montet: ses rapports avec les ateliers du GrandPressigny ", Bulletin des amis du musée de Préhistoire du Crand-Pressigny, 49, p. 37-42.

Hamon T., Irribaria R., Rlalland Y., VERJUX C.

1997 : "Le groupe de Chambon à la lumière des découvertes récentes en région Centre ", in La Culture de Cerny. Nouvelle économie, nouvelle société au Néolithique. Actes du colloque international de Nemours 1994, Nemours, éd. APRAIF (Mémoires du musée de Préhistoire d'île-de-France, 6), p. 195-217.
HONEGGER M.

2001 : L'industrie lithique taillée du Néolithique moyen et final de Suisse, Paris, CNRS Éditions (coll. CRA Monographies, 22), $354 \mathrm{p}$.

2002 : "Les influences méridionales dans les industries lithiques du Néolithique suisse ", in Les industries lithiques taillees holocènes du Bassin rhodanien: problèmes et actualités, Actes de la table ronde de Lyon, 8-9 déc. 2000, éd. Monique Mergoil, p. $135-147$.

\section{HutTunen P.}

1980 : " Early land use, especially the slashand-burn cultivation in the commune of Lammi, southern Finland, interpreted mainly using pollen and charcoal analyses ", Acta Botanica Fennica, 113, p. $1-45$.

INNES J. B., SIMMONS I. G.

2000 : "Mid-Holocene charcoal stratigraphy, fire history and Palaeoecology at North Gill, North York Moors, UK ", Palaeogeography Palaeoclimatology Palaeoecology, 164, 1-4, p. 151-166.

\section{IRRIBARIA $\mathbf{R}$.}

1997 : " Comparaison céramologique du Cerny et du Chambon ", in La Culture de Cerny. Nouvelle économie, nouvelle société au Néolithique, actes $d u$ colloque international de. Nemours 1994, Nemours, éd. APRAIF (Mémoires du musée de Préhistoire d'îlede-France, 6), p. 219-238.

IVERSEN J.

1941 : " Land occupation in Denmark's Stone Age ", Danmarks Geologiske Undersøgelse, II, p. $66-68$.

1949: "The influence of Prehistoric Man on Vegetation ", Danmark's Geologiske Undersøgelse, IV, 3, 6, p. 5-25.

1956 : "Forest clearance in the Stone Age ", Scientific American, 194, p. 36-41.

Krausz S., Constantin G.

1995 : "Un site d'habitat de la culture d'Artenac à Moulins-sur-Céphons (Indre) ", Bulletin de la Société préhistorique française, 92, 3, p. 346-352.

\section{LABLANCHE G., DePrez N.}

1984 : Carte géologique et notice explicative de la feuille Châteauneuf-sur-Cher au 1/50000, Orléans, éd. du BRGM.

\section{LE JEUNE $\mathbf{Y}$.}

1997 : Étude de l'érosion des sols sur la rive occidentale du lac de Chalain (Jura) : une manière 
d'appréhender l'histoire des paysages, mém. de DEA “ Environnement et Archéologie ", Paris, Muséum national d'histoire naturelle, $75 \mathrm{p}$.

Mackuin M. G., Bonsall C., Davies F. M., RoBINSON M. R.

2000 : " Human-environment interactions during the Holocene : new data and interpretations from the Oban area, Argyll, Scotland ", The Holocene, 10, 1, p. 109-121.

\section{MaGnY M.}

1992 : " Holocene lake-level fluctuations in Jura and the northern subalpine ranges, France: regional pattern and climatic implications ", Boreas, 21, p. 319-334.

1995 : "Successive Oceanic and Solar Forcing Indicated by Younger Dryas and Early Holocene Climatic Oscillations in the Jura ", Quaternary Research, 43, p. 279-285.

1998 : « Reconstruction of Holocene lake-level changes in the French Jura : methods and results ", in BuRkhard F., Frfuzel. B. ET AL., Palaeohydrology as reflected in lake-level changes as climatic evidence for Holocene times, European Palaeoclimate and Man 17, ESF Project, Sonderdruck, Stuttgart, G. Fischer, p. 68-85.

1999 : "Lake-level fluctuations in the Jura and French subalpine ranges associated with ice-rafting events in the North Atlantic and variations in the polar atmospheric circulation ", Quaternaire, 10, 1, p. 61-64.

Malenfant M., Cauvin M.-C., Chaffenet G.

1971 : "Découverte d'une industrie macrolithique récente de faciès pressignien à Vassieux-en-Vercors (Drôme) ", Comptes rendus de l'Académie des sciences, 272, p. 1491-1494.

\section{MaLlet N.}

1992 : « Le Grand-Pressigny : ses relations avec la civilisation Saône-Rhône ", Suppl. au Bulletin de la Société des amis du musée du Grand-Pressigny.

Maluet N. avec la collab. de Richard G., Genty P., Delcourt-Vlaeminck M.

2000 : " La diffusion des silex du GrandPressigny au Néolithique final ; état actuel de l'inventaire ", Bulletin des amis du musée de Préhistoire du Grand-Pressigny, 51, p. 27-31.

Mallet N., Richard G., Genty P., Verjux C. 2004 : "La diffusion des silex du GrandPressigny dans le Bassin parisien ", in VAN DF.R LiNde. M., Salanova L. (DIR.), Le troisième millénaire dans le nord de la France et en Belgique, Actes de la journée d'études SRBAP-SPF, 8 mars 2003, Lille, Mémoires de la Société préhistorique française, $\mathrm{XXXV}$, Anthropologica et praehistorica, Bulletin de la société royale belge d'anthropologie et de préhistoire, 115, p. 123-138.

\section{MARQuet J.-C., Millet-Richard L.-A.}

2000 : Les sites préhistoriques de la Touraine du Sud, Chambray-les-Tours, éd. ARCHEA (coll. Cahiers de la Claise tourangelle).

\section{MAZOYER M., ROUDART L.}

1997: Histoire des agricultures $d u$ monde: $d u$ Néolithique à la crise contemporaine, Paris, Seuil, $545 \mathrm{p}$.

\section{MÉTAILIÉ J.P.}

1981 : Le feu pastoral dans les Pyrénées centrales (Barousse, Oueil, Larboust), Paris, ćd. du CNRS, 195 p.

\section{MiLCENT P.-Y.}

1999 : Recherches sur le premier âge du Fer en France centrale, thèse de doctorat, univ. Paris-I - Panthéon-Sorbonne, $836 \mathrm{p}$

Millspaugh S. H., Whitlock C. BaRTLEIN P. J.

2000 : "Variations in fire frequency and climate over the past $17000 \mathrm{yr}$ in central Yellowstone National Park ", Geology, 28, 3, 211-214.

Mohr J. A., Whitlock C., Skinner C. N.

2000 : "Postglacial vegetation and fire history, eastern Klamath Mountains, California, USA ", The Holocene, 10, 4, p. 587-601.

\section{MOORE J}

2000 : « Forest fire and human interaction in the early Holocene woodlands of Britain ", Palaeogeography Palaeoclimatology Palaeoecology, 164, 1-4, p. 125-137.

MoRdant C., RICHARD A. (Éd.)

1993 : L'habitat et l'occupation du sol à l'âge du Bronze en Europe, Paris, éd. du Comité des travaux historiques et scientifiques (coll. Documents préhistoriques, 4 ).

\section{MORISON K. D.}

1994 : « Monitoring regional fire history through size-specific analysis of microscopic charcoal : the last 600 years in South India ", Journal of Archeological Science, 21, p. $675-685$.

Pétrequin P.

1994 : “ Fontenu Les Vernois P12 ", Bilan scientifique régional de Franche-Comté, DRAC/SRA, p. 69-70.
Pétrequin P. (DIR.)

1997 : Les sites littoraux néolithiques de Clairvauxles-Lacs et de Chalain (Jura), -III- Chalain station 3, 3200-2900 ax. J.-C., 2, Paris, éd. de la Maison des sciences de l'Homme, $765 \mathrm{p}$.

Pétrequin P., ARbogast R.-M., BourquinMignot C., Lavier C. Viellet A.

1998 : « Demographic growth, environmental changes and technical adaptations: responses of an agricultural community from the $32^{\text {nd }}$ to the $30^{\text {th }}$ centuries $\mathrm{BC}$ ", World Archaeology, 30, 2, p. 181-192.

\section{Pitkänen A., Huttunen P.}

1999 : " A 1300-year forest-fire history at a site in eastern Finland based on charcoal and pollen records in laminated lakes sediment ", The Holocene, 9, 3, p. 311-320.

Plisson H., Mallet N., Bocquet A., RAMSEYER D.

2002 : «Utilisation et rôle des outils en silex du Grand-Pressigny dans les villages de Charavines et de Portalban (Néolithique final) ", Bulletin de la Société préhistorique française, 99, 4, p. 793-811.

\section{Provensal M., Morhange C.}

1994 : "Seuils climatiques et réponses morphogéniques en Basse-Provence à l'Holocène ", Colloque AFEQ Échelles des variations chronoclimatiques quaternaires et réponses des environnements, Quaternaire, 5, 3-4, p. 113-118.

\section{ReNAULT S.}

1998 : "Économie de la matière première. L'exemple de la production, au Néolithique final en Provence, des grandes lames en silex zoné oligocène du bassin de Forcalquier (Alpes-de-HauteProvence) ", in D'ANvi A.,BINder D. (DIR.), Rencontres méridionales de Préhistoire récente, 2 session, Arles, 8-9 nov. 1996, Antibes, éd. APDCA, p. 145-161.

\section{RICHARD H.}

1994 : "Indices polliniques d'une néolithisation précoce sur le premier plateau du Jura (France) ", Comptes rendus de l'Académie des sciences de Paris, Série IIa, Sciences de la terre et des planètes, p. 993-999.

1995 : « Indices polliniques d'anthropisation dans les diagrammes polliniques du massif jurassien ", Palynosciences, 3, p. $37-49$.

2000: « L'introduction de l'agriculture sur la montagne jurassienne. Plus d'un millé- 
naire de succès et d'échecs apparents ", in Guilanis. J., "La très longue durée ", Etudes rurales, 153-154, p. 115-125.

\section{Richard H., Ruffaldi P.}

1996 : "L'hypothèse du déterminisme climatique des premières traces polliniques de néolithisation sur le massif jurassien (France) ", Comptes rendus de l'Académie des sciences de Paris, Série IIa, Sciences de la terre et des planètes, 322, p. 77-83.

\section{Richoz I., Gaillard M.-J., Magny M.}

$1994:$ : The influence of human activities and climate on the development of vegetation at Seedorf, Southern Swiss Plateau during the Holocene : a case study ", Dissertationes Botanicce, 234, p. 423-445.

\section{Sigaut F.}

1975 : L'Agriculture et le feu. Rôle el place du feu dans les techniques de préparation du champ de I'ancienne agriculture européenne, Paris, EHESS / La Haye, Mouton \& Co (coll. Cahiers des études rurales, 1), $320 \mathrm{p}$.

Stutver M., Reimer P. J., Bard E., Beck J. W., Burr G. S., Hughen K. A., Kromer B., McCormac F. G., Plicht J., Spurk M.

1998 : « INTCAL 98 Radiocarbon age calibration 24,000-0 cal. BP ", Radiocarbon, 40, 3, p. $1041-1083$.

Tinner W., Hubschmid P., Wehrla M., ammann B., Conedera M.

1999 : "Long-term forest fire coology and dynamics in southern Switzerland ", Journal of Ecology, 87, 2, p. 273-289.

VAN DER WAALS J.-D.

1991 : "Silex du Grand-Pressigny aux PaysBas ", in Desprite. J., Verju x C., Pieloole J.
IIT Al.., La Région Centre: carrefour d'influences? Actes du $14^{\prime \prime}$ colloque interrégional sur le Néolithique, Blois, 16-18 oct. 1987, Suppl. au Bulletin de la Société archéologique el littéraire du Vendômois, p. 193-200.

Van Geel B., Raspopov O. M., Renssen H., Van der Plicht J., Dergachey V. A, MEIJER H. A. J.

1999 : " The role of solar forcing upon climate change ", Quaternary Science Reviews, 18, 3, p. $331-338$.

\section{VANNIÈRE B.}

2001 : Feu, agro-pastoralisme et dynamiques environnementales en France durant l'Holocène. Analyse du signal incendie, approches sédimentologiques et étude de cas en Berry, Pyrénées et Franche-Comté, thèse de doctorat, Institut national agronomique de Paris-Grignon, $329 \mathrm{p}$.

2004 : "Les micro-charbons: indicateur paléoenvironnemental de l'anthropisation. Exemple d'étude en contexte alluvial (Vierzon, Cher) ", in Actualite de la recherche en histoire et archéologie agraires, Actes du colloque international AGER V, sept. 2000, Besangon, Presses universitaires franc-comtoises, Annales littéraires, $\mathrm{X}$, Série “ Environnement, sociétés et archéologie ", 5, p. 283-299.

VANNiÈre B., Bossuet G., WalterSimonet A.-V., Gauthier E., Barral P., Petit C., Buatier M., Daubigney A.

2003 : " Land use change, soil erosion and alluvial dynamic in the lower Doubs Valley over the $1^{\text {st }}$ millenium AD (Neublans, Jura, France) ", Journal of Archaeological Science, 30, p. 1283-1299.
Vannière B., Galop D., Rendu C., Davasse B. 2001 : " Feu et pratiques agro-pastorales dans les Pyrénées Orientales: le cas de la montagne d'Enveig (Cerdagne, France) ", Sud-Ouest européen, 11, p. 29-42.

\section{Vannière B., Laggoun-Defarge $F$.}

2002 : «Première contribution à l'étude des évolutions paléohydrologiques et à l'histoire des feux en Champagne berrichonne durant l'Holocène. Le cas du "Marais du Grand-Chaumet" (Indre, France) ", in BRAVARI) J.-P., MAGNY M. (DIR.), Les Fleuves ont une histoires: paléoenvironnement des rivières et des lacs fransais depuis 15000 ans, éd. Errance, p. 101-124.

\section{VERJUX C.}

1999 : "Chronologie des rites funéraires mésolithiques à Auneau (Eure-et-Loir, France) ", in ThÉveNin A., BINT\% P. (DIR.) L'Europe des derniers chasseurs. Peuplement et parlementairement de l'Épipaléolithique et du Mésolithique: Actes du $V^{p}$ colloque international UISPP, commission XII, Crenoble, 18-23 sept. 1995, Paris, Documents préhistoriques, 12, p. 293-302.

\section{VILLES A.}

2003 : "Que savons-nous des affinités culturelles du Grand-Pressigny au Néolithique récent et final ?", Bulletin des amis du musée de Prêhistoire du Grand-Pressigrny, 54, p. 43-74.

\section{VUORELA I.}

1986 : "Palynological evidence of slash-andburn cultivation in South Finland ", in BFHRE. K. E. (E.D.), Anthropogenic indicators in pollen diagrams, Rotterdam, Balkema, p. 53-64. 